\title{
THE PICARD GROUP OF NONCOMMUTATIVE RINGS, IN PARTICULAR OF ORDERS $\left({ }^{1}\right)$
}

BY

\section{A. FRÖHLICH}

ABSTRACT. The structure of the Picard group of not necessarily commutative rings, and specifically of orders, and its relation to the automorphism group are studied, mainly with arithmetic applications in mind.

This paper is concerned with the Picard group, $\mathrm{Pic}(A)$, of a noncommutative (i.e. not necessarily commutative) ring $A$ with identity, defined via the tensor product of invertible bimodules (see e.g. [B1] or [B2]). Although some general results on $\mathrm{Pic}(A)$ are known, relatively little systematic work has been done so far. The real interest of the noncommutative theory has until now been the special case of Azumaya (central separable) algebras, when in fact Pic $(A)$ coincides with $\mathrm{Pic}$ of the commutative ring cent $(A)$ (the centre of $A$ ) (see [RZ]). On the other hand our principal interest lies in orders. But although our strongest results come in this case, some of these extend without too much weakening to a wider class of rings. In fact in the early part of the paper the treatment is completely general.

Although most of the present paper is algebraic in spirit, the whole work was done with applications to the arithmetic of noncommutative orders over the ring $\mathbf{Z}$ of integers in mind. Our theory then becomes a tool both for certain local as well as for noncommutative "local-global" problems, and it leads up to the consideration of arithmetic subgroups in certain algebraic groups. Moreover the explicit computations for integral group rings given at the end of this paper are really number theoretic, i.e. depend crucially on $\mathrm{Z}$ being the base ring.

For the general theory one can, without loss of generality, view the ring $A$ as an algebra over some commutative ring $R$ and look at $\operatorname{Pic}_{R}(A)$, the group given by bimodules with $R$ acting the same on both sides. One can always take $R=\mathrm{Z}$, noting that $\mathrm{Pic}(A)=\mathrm{Pic}_{\mathrm{Z}}(A)$. For theoretical reasons and for applications it is however the normal subgroup $\operatorname{Pic}_{\text {cent }(A)}(A)=\operatorname{Picent}(A)$ which is the really interesting object, even when one considers $R$-orders, and $R \neq$ cent $(A)$.

Received by the editors July 19, 1972.

AMS (MOS) subject classifications (1970). Primary 16A54, 16A18, 16A26, 16 A72.

(1) Most of this work was done while the author was Visiting Professor in the University of Arizona at Tucson and was partly supported by the National Science Foundation. 
The significance of the Picard group lies in its relation to other aspects of ring theory. We mention three of these.

1. Multiplicative ideal theory. If $A$ is an order over an integral domain $R$ in a finite-dimensional semisimple $K$-algebra, $K$ the quotient field of $R$, then Picent $(A)$ is a quotient of the group $I(A)$ of invertible fractional ideals, just as in the commutative case. We shall show in fact that this is a special case of a completely general exact sequence, associated with a change of base ring $R$, for any $R$-algebra $A$. This is based on an adaptation of $K$-theory techniques to "noncommutative" products.

2. The class group of rank one projectives. This group, denoted by $C(A)$, is defined for orders $A$, via the direct sum. In the commutative theory one gets an isomorphism Picent $(A) \cong C(A)$. There is however also a connection in the noncommutative case, and in a subsequent joint note [FRU] we shall for certain orders $A$, on the basis of results of the present paper, define a homomorphism Picent $(A) \rightarrow C(A)$ with interesting properties.

3. The automorpbism group of the algebra $A$. There is a homomorphism Aut $_{R}(A) \rightarrow \mathrm{Pic}_{R}(A)$ (cf. [RZ], [B1]) which gives rise to a homomorphism $\Omega$ : Autcent $(A) \rightarrow$ Picent $(A)$, where Autcent $(A)$ consists of those algebra automorphisms of $A$ which leave the centre elementwise fixed. This is the most interesting and specifically noncommutative aspect of the theory. We shall derive a significant variant of $\Omega$, useful in applications, by considering algebras $A \otimes_{C} E$, $C=$ cent $(A)$, with $E$ running over $C$-Azumaya algebras or just over matrix rings, and then going to the limit.

The role of the Picard group in its relation to other objects explains why one should want to study this group. The main purpose of this paper is to show how one can study it. We shall introduce a sequence of normal subgroups and analyse the consecutive factors. In the case when $A$ is an order over a Dedekind domain $R$, in a separable algebra over the quotient field $K$, we shall obtain a fairly complete description which makes explicit computations feasible. We display the decreasing subgroups (on the left) and the quotients (on the right) in a diagram, where $C=\operatorname{cent}(A)$.

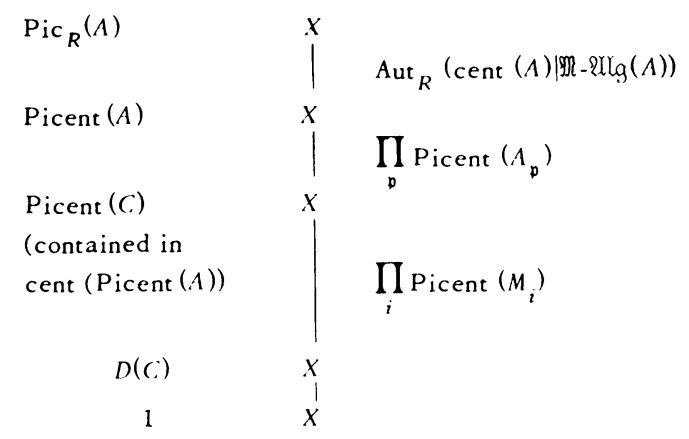


Explanations. (i) That $\operatorname{Pic}_{R}(A) / \operatorname{Picent}(A)$ is a subgroup of $A_{\text {ut }}$ (cent $(A)$ ) (algebra automorphisms) has been known. Here we shall determine this subgroup, as that consisting of those automorphisms which fix the Morita class of $A$ over cent $(A)$. All this applies not only to orders, but in complete generality. In concrete situations this group is easily computed. (ii) The product $\Pi_{\mathfrak{p}}$ runs over the maximal ideals $p$ of $R, A_{\mathfrak{p}}$ denoting localisations or completions. The exact (and central) sequence

$$
1 \rightarrow \text { Picent }(C) \rightarrow \text { Picent }(A) \rightarrow \prod_{\mathfrak{p}} \text { Picent }\left(A_{p}\right) \rightarrow 1
$$

is really the central tool in the analysis and computation of $\mathrm{Pic}_{R}(A)$. Moreover, for almost all $\mathfrak{p}$, Picent $\left(A_{\mathfrak{p}}\right)=1$, thus the product is finite. The sequence $(*)$ remains exact, except for the right hand bit " $\rightarrow 1$ ", under much more general hypotheses. (iii) For the local (or semilocal) case, i.e. for the description of the factor Picent $\left(A_{\mathfrak{p}}\right)$ we shall develop basic reduction theorems. (iv) In the commutative case one has a fairly effective theory (see e.g. [F]). The $M_{i}$ are the maximal orders in the extension fields of $K$ which occur as factors in the commutative $K$-algebra spanned by $C$, and $D(C)$ has a nice description in terms of unit groups.

The last third of the paper is taken up with particular orders and with computations. These are included, being of independent interest in themselves, while at the same time illustrating and testing the general theory. In particular the explicit calculations-although at first sight rather special-bring out genuine number theoretic aspects, and in fact I believe that one of the lines, along which a good theory of orders should develop, is arithmetic specialisation.

Not included in this paper is a systematic treatment of fibre products. I have also not considered special classes of algebras which are still somewhat more general than orders over a Dedekind domain in a semisimple algebra (e.g. $R$ a Krull domain, or nonsemisimple algebras etc.).

I wish to record my gratitude to I. Reiner and S. Ullom for careful checking and constructive criticism of a first draft, which led to improvements and to the elimination of some errors.

1. The Picard group and automorphisms. All rings have identities. These act as identity maps on all modules and are preserved by ring homomorphisms.

Let $A$ and $B$ be rings. An $A-B$-bimodule ${ }_{A} X_{B}$ ( $A$ acting on the left, $B$ on the right) is invertible if it satisfies the following conditions (I) and (II), each of which implies the other.

(I) $X_{B}$ is finitely generated projective (i.e. $X$ as $B$-module), and $A \cong$ End $\left(X_{B}\right)$ via the action on $X$, plus analogous statements on interchange of $A$ and $B$. 
(II) There is a bimodule ${ }_{B} Y_{A}$ and isomorphisms

$$
\begin{array}{ll}
f: Y \bigotimes_{A} X \cong B & \text { (of } B \text { - } B \text {-bimodules) } \\
g: X \bigotimes_{B} Y \cong A & \text { (of } A-A \text {-bimodules) }
\end{array}
$$

so that the diagrams

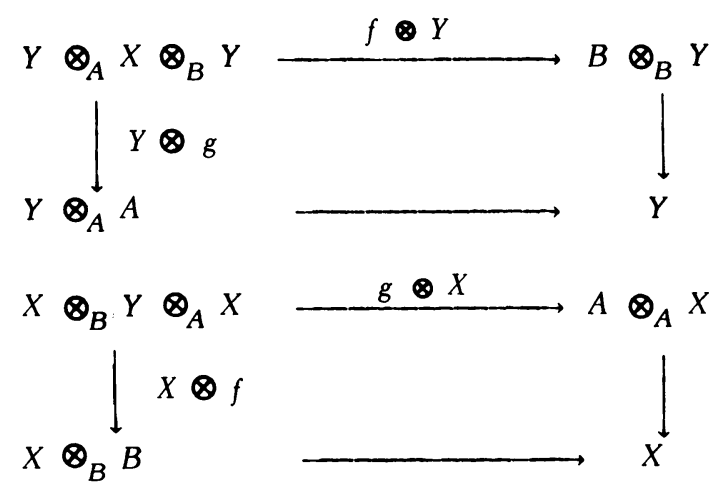

commute.

In (II) $X$ will determine its inverse $Y$, and the maps $f$ and $g$ uniquely to within isomorphism. For these concepts see e.g. [B1] or [B2].

Now suppose $A$ and $B$ to be $R$-algebras, $R$ being a commutative ring. If the $R$-module structures of ${ }_{A} X_{B}$, via $A$ and via $B$, coincide then we shall call $X$ an $A$-B-bimodule over $R$. The isomorphism classes $(X)$ of invertible $A \cdot A$-bimodules under the product

$$
\left(X_{1}\right)\left(X_{2}\right)=\left(X_{1} \otimes_{A} X_{2}\right)
$$

form the Picard group, $\mathrm{Pic}(A)$, of $A$, those "over $R$ " a subgroup $\mathrm{Pic}_{R}(A)$. Of course $\mathrm{Pic}(A)=\mathrm{Pic}_{\mathrm{Z}}(A)$, where $\mathrm{Z}$ is the ring of integers.

We wish to compare $\mathrm{Pic}_{R}(A)$ and the group of algebra automorphisms of $A$ over $R$, which we shall denote by $\operatorname{Aut}_{R}(A)$. For a proper understanding of the situation it is worth introducing two categories, $\operatorname{llg}_{R}$ and $\mathbb{M}_{-} \mathscr{U M g}_{R}$. The objects of both are the $R$-algebras, the morphisms of $\operatorname{llg}_{R}$ are the isomorphisms of $R$-algebras, those of $M-2 \mathrm{Mg}_{R}$ are the Morita equivalences over $R$. A Morita equivalence $B \sim A$ over $R$ is simply an isomorphism class $(M)$ of invertible $A$-B-bimodules ${ }_{A} M_{B}$ over $R$. If $(N)$ is a Morita equivalence $C \sim B$, then the compositum is given by $\left(M \otimes_{B} N\right)$. Thus $\operatorname{Aut}_{R}(A)$ and $\mathrm{Pic}_{R}(A)$ are the automorphism groups of the object $A$ in $\operatorname{llg}_{R}$, and in $\mathscr{M}_{-} 2 \mathrm{Mg}_{R}$, respectively. In particular $\mathrm{Pic}_{R}$ is a functor from the category of Morita equivalences of $R$-algebras to the category of groups and isomorphisms.

Both $\mathscr{L M g}_{R}$ and $\mathfrak{M}_{-} 2 \mathrm{Mg}_{R}$ are categories with product in the sense of [BI], given by $\otimes_{R}$. The product of objects $A_{1}$ and $A_{2}$ is $A_{1} \otimes_{R} A_{2}$. The product of 
morphisms $f_{i}: B_{i} \rightarrow A_{i}(i=1,2)$ in $2 \mathrm{Ulg}_{R}$ is $f_{1} \otimes_{R} f_{2}$, the product of Morita equivalences $\left(M_{i}\right): B_{i} \sim A_{i}$ is $\left(M_{1} \otimes_{R} M_{2}\right)$. The crucial point here is that if $M_{i}$ is an invertible $A_{i}-B_{i}$-bimodule over $R$ then $M_{1} \otimes_{R} M_{2}$ is an invertible $A_{1} \otimes_{R} A_{2}-B_{1} \otimes_{R} B_{2}$-bimodule. In particular we get homomorphisms

$$
\begin{aligned}
& \operatorname{Pic}_{R}\left(A_{1}\right) \times \operatorname{Pic}_{R}\left(A_{2}\right) \rightarrow \operatorname{Pic}_{R}\left(A_{1} \otimes_{R} A_{2}\right), \\
& \operatorname{Aut}_{R}\left(A_{1}\right) \times \operatorname{Aut}_{R}\left(A_{2}\right) \rightarrow \operatorname{Aut}_{R}\left(A_{1} \otimes_{R} A_{2}\right),
\end{aligned}
$$

the first of which is compatible with Morita equivalence, and so in particular homomorphisms

$$
\begin{aligned}
& \operatorname{Pic}_{R}\left(A_{1}\right) \rightarrow \operatorname{Pic}_{R}\left(A_{1} \bigotimes_{R} A_{2}\right), \\
& \operatorname{Aut}_{R}\left(A_{1}\right) \rightarrow \operatorname{Aut}_{R}\left(A_{1} \bigotimes_{R} A_{2}\right),
\end{aligned}
$$

given by $(X) \mapsto\left(X \otimes_{R} A_{2}\right)$, and by $f \mapsto f \otimes_{R} A_{2}$ respectively. If in particular $A_{2}$ is an $R$-Azumaya algebra (central separable $R$-algebra), then it is an object of $\Re-\mathrm{MLg}_{R}$ invertible under the product $\otimes_{R}$ and hence, using the theory of categories with product, one deduces that the first map in (1.2) is an isomorphism.

We now have a functor

$$
\Omega: \operatorname{lug}_{R} \rightarrow M_{-2} \operatorname{Ug}_{R} \text {, }
$$

which is the identity on objects and associates with an isomorphism $f: B \rightarrow A$ of $R$-algebras the class of the invertible $A$-B-bimodule $A_{f}$. Here we use the following notation: If $X$ is an $A_{1}-A_{2}$-bimodule and $g: B_{1} \rightarrow A_{1}, f: B_{2} \rightarrow A_{2}$ are algebra homomorphisms then ${ }_{g} X$ is an isomorphic copy of the additive group of $X$ under a map $x \rightarrow g_{f}^{x}$, made into a $B_{1}-B_{2}$-bimodule by setting

$$
b_{1}\left({ }_{g} x_{f}\right) b_{2}={ }_{g}\left(g\left(b_{1}\right) x f\left(b_{2}\right)\right), \quad \forall x \in X, b_{1} \in B_{1}, b_{2} \in B_{2} .
$$

If one of the two maps $g$ and $f$ is an identity map 1 , then we shall write ${ }_{1} X_{f}=$ $X_{f}$ or ${ }_{g} X_{1}={ }_{g} X$. The verification that $\Omega$ is indeed a functor and that it preserves the product $\otimes_{R}$ is now routine.

In preparation for our first theorem we introduce some more notations. $\operatorname{In}(A)$ is the group of inner automorphisms $a \mapsto b a b^{-1}$ of $A$, a subgroup of $\operatorname{Aut}_{R}(A)$. $\operatorname{Proj}(A)$ is the set of isomorphism classes of finitely generated faithfully projective left $A$-modules. Forgetting the right action of $A$ yields a map

$$
\gamma: \operatorname{Pic}(A) \rightarrow \operatorname{Proj}(A) \text {. }
$$

Moreover $\operatorname{Pic}(A)$ acts on $\operatorname{Proj}(A)$ by

$$
(X),\{Y\} \mapsto\left\{X \otimes_{A} Y\right\}, \quad(X) \in \operatorname{Pic}(A),\{Y\} \in \operatorname{Proj}(A) .
$$

Next observe that associated with a pair $E_{1}, E_{2}$ of $R$-Asumaya algebras we get a homomorphism $F\left(A \otimes_{R} E_{1}\right) \rightarrow F\left(A \otimes_{R} E_{1} \otimes_{R} E_{2}\right)$ for $F=\mathrm{Pic}_{R}$, Aut ${ }_{R}$ 
(cf. (1.3)) and similarly for $F=I n .^{*}$ These homomorphisms satisfy, for varying $E_{1}, E_{2}$, the obvious compatibility conditions, allowing us to define limit groups $\underset{\lim }{\rightarrow} F\left(A \otimes_{R} E\right)$. For $F=\mathrm{Pic}_{R}$, the homomorphisms are all bijective (recall the remark made after (1.2)). Hence we may identify $\underset{\lim }{\rightarrow} \operatorname{Pic}_{R}\left(A \otimes_{R} E\right)=\operatorname{Pic}_{R}(A)$.

Theorem 1. (i) The sequence

$$
\left(S_{A}\right): 1 \rightarrow \operatorname{In}(A) \stackrel{\iota}{\rightarrow} \operatorname{Aut}_{R}(A) \stackrel{\boldsymbol{\Omega}}{\rightarrow} \mathrm{Pic}_{R}(A)
$$

is exact, and the left cosets in $\operatorname{Pic}_{R}(A) / \operatorname{Im} \Omega$ are the fibres of the map $\gamma$ : $\operatorname{Pic}_{R}(A) \rightarrow \operatorname{Proj}(A)$. The orbits of $\operatorname{Proj}(A)$ under the action of $\mathrm{Pic}_{R}(A)$ are the fibres of the map which sends $\{Y\} \in \operatorname{Proj}(A)$ into the R-algebra isomorphism class of $\operatorname{End}_{A}(Y)$.

Moreover, $\Omega$ is compatible with maps (1.1), and analogously for $\iota$ and $\gamma$.

(ii) For $(X),(Y) \in \mathrm{Pic}_{R}(A)$ and $M$ a faitbful, finitely generated projective $R-$ module,

$$
X \otimes_{R} \operatorname{End}_{R}(M) \cong Y \otimes_{R} \operatorname{End}_{R}(M)
$$

(isomorphism of left $A \otimes_{R} \operatorname{End}_{R}(M)$-modules) if and only if

$$
X \otimes_{R} M^{*} \cong Y \otimes_{R} M^{*}
$$

(isomorphism of left A-modules), where $M^{*}=\operatorname{Hom}_{R}(M, R)$.

(iii) By going to the limit over the R-Azumaya algebra $E$, the sequences $\left(S_{A \otimes E}\right)$ yield an exact sequence

$$
\left(\bar{S}_{A}\right): 1 \rightarrow \stackrel{\lim }{\longrightarrow} \ln \left(A \otimes_{R} E\right) \stackrel{\grave{\imath}}{\rightarrow} \underset{\longrightarrow}{\lim } A_{\mathrm{ut}}\left(A \otimes_{R} E\right) \stackrel{\overline{\mathbf{\Omega}}}{\rightarrow} \mathrm{Pic}_{R}(A) .
$$

Moreover, the following conditions are equivalent:

(a) $(X)=(Y)(U),(U) \in \operatorname{Im} \bar{\Omega}$.

(b) $X \otimes_{R} E \cong Y \otimes_{R} E$ (isomorpbism of left $A \otimes_{R} E$-modules) for some $E$ (Azumaya over $R$ ).

(c) $X \otimes_{R} N \cong Y \otimes_{R} N$ (isomorpbism of left A-modules) for some faithful finitely generated projective $R$-module $N$.

(d) $X^{(n)} \cong Y^{(n)}$ (isomorphism of left A-modules) for some $n>0$, where $V^{(n)}$ is the direct sum of $n$ copies of $V$.

Remark. For the sequence $\left(S_{A}\right)$ in (i) and a characterisation of $\mathrm{Pic}_{R}(A) / \operatorname{Im} \Omega$ see [RZ] (with $A$ restricted to be Azumaya) and [B1]. The connection between automorphisms and the Picard group was for the first time pointed out in [RZ].

Proof of Theorem 1. (i) Let $f \in$ Aut $_{R}(A), a \mapsto a_{f}$ is an isomorphism $A \rightarrow$ $A_{f}$ of left $A$-modules, and so every such isomorphism $g: A \rightarrow A_{f}$ is of form $g(a)=(a b)_{f}$, where $b$ is some unit of $A$. Now one computes 


$$
g(a c)=g(a) f^{-1}\left(b^{-1} c b\right), \quad \forall a, c \in A .
$$

Hence $g$ is an isomorphism of $A$-A-bimodules if and only if $b^{-1} c b=f(c), \forall c \in A$. We have thus shown that indeed $\operatorname{In}(A)=\operatorname{Ker} \Omega$.

Next observe that for any $A$-A-bimodule $X, X \otimes_{A} A_{f}=X$ is isomorphic with $X$ as left $A$-module. Conversely if $X$ and $Y$ are invertible $A-A$-bimodules, and $b: X \cong Y$ is an isomorphism of left $A$-modules then the compositum

$$
A \stackrel{t^{y}}{\longrightarrow} \operatorname{End}\left({ }_{A} Y\right) \stackrel{h^{*}}{\longrightarrow} \text { End }\left({ }_{A} X\right) \stackrel{t^{-1}}{\longrightarrow} A,
$$

where $t_{Y}, t_{X}$ are given by the right $A$-module structure, is an automorphism $f$ of $A$ so that $b(x f(a))=b(x) a$. Thus indeed $Y \cong X_{f}$ as $A-A$-bimodule. We have now shown that the cosets in $\operatorname{Pic}_{R}(A) / \operatorname{Im} \Omega$ are the fibres of $\gamma$.

The fact that $\Omega$ preserves products has already been noted. That the same is true for $\iota$ and $\gamma$ is obvious.

(ii) We are viewing $M^{*}$ as left $E$-module, for $E=\operatorname{End}_{R}(M)$. Then $M^{*}$ is an invertible $R \cdot E$-bimodule, hence $A \otimes_{R} M^{*}$ is an invertible $A \cdot A \otimes_{R} E$-bimodule, defining a bijection of the set of isomorphism classes $\{V\}$ of left $A \otimes_{R} E$-modules onto the set of isomorphism classes $\left\{\left(A \otimes_{R} M^{*}\right) \otimes_{(A \otimes E)} V\right\}$ of left $A$-modules. In this the isomorphism class of $U \otimes_{R} E$ with $U$ a left- $A$-module, corresponds to that of $U \otimes_{R} M^{*}$.

(iii) By (i), using the fact that $\iota, \Omega$ and $\gamma$ preserve products, we obtain commutative diagrams

$$
\begin{aligned}
& I n\left(A \otimes E_{1}\right) \longrightarrow A_{R}\left(A \otimes E_{1}\right) \longrightarrow \operatorname{Pic}_{R}\left(A \otimes E_{1}\right) \longrightarrow \operatorname{Proj}\left(A \otimes E_{1}\right) \\
& I n\left(A \otimes E_{1} \otimes E_{2}\right) \rightarrow A u t_{R}\left(A \otimes E_{1} \otimes E_{2}\right) \rightarrow \operatorname{Pic}_{R}\left(A \otimes E_{1} \otimes E_{2}\right) \rightarrow \operatorname{Proj}\left(A \otimes E_{1} \otimes E_{2}\right) \\
& \left(\otimes=\otimes_{R}\right) \text {. Note in passing that, as } E_{2} \text { is a faithful projective } R \text {-module, the } \\
& \text { column of Aut }{ }_{R} \text { is injective, hence so is that of } I n . \text { Going to the limit, we get } \\
& \text { the exact sequence }\left(\bar{S}_{A}\right) \text { and the equivalence of (a) and (b), all from part (i) of } \\
& \text { the theorem. }
\end{aligned}
$$

Moreover, condition (b) is equivalent with the similar condition where $E$ is restricted to algebras $\operatorname{End}_{R}(M)$ with $M$ faithful, finitely generated projective over $R$-as can be seen by tensoring with $E^{\text {op }}$ in (b). But now one concludes from part (ii) that (b) is equivalent with (c). Finally, (d) is condition (c) with $N$ restricted to $R^{(n)}$, for $n>0$, thus (d) implies (c). Conversely, if (c) holds, tensor both sides with a module $M$ so that $N \otimes_{R} M \cong R^{(n)}$, and we get (d). This completes the proof of the theorem.

The ring $A$ may be viewed as an algebra over its centre cent $(A)$. We define the central Picard group of $A$ to be 


$$
\operatorname{Pic}_{\text {cent }(A)}(A)=\text { Picent }(A) \text {. }
$$

If $A$ is an $R$-algebra then $\mathrm{Picent}(A)$ is a subgroup of $\mathrm{Pic}_{R}(A)$. This central Picard group will play an important role in the sequel. Here we note some consequences of the preceding theorem, relating to it. Side by side with Picent $(A)$ we shall also have to consider the central automorphism group

$$
\operatorname{Aut}_{\operatorname{cent}(A)}(A)=\operatorname{Autcent}(A) .
$$

Note that in the definition of these two groups the commutative base ring itself depends on $A$.

With these definitions we get

Corollary. We bave exact sequences

$$
1 \rightarrow \operatorname{In}(A) \rightarrow \text { Autcent }(A) \stackrel{\mathfrak{\Omega}}{\rightarrow} \text { Picent }(A)
$$

and (with $C=\operatorname{cent}(A))$

$$
1 \rightarrow \underset{\lim }{\rightarrow} \ln \left(A \otimes_{C} E\right) \rightarrow \underset{\lim }{\rightarrow} \text { Autcent }\left(A \otimes_{C} E\right) \stackrel{\overline{\mathbf{\Omega}}}{\rightarrow} \text { Picent }(A),
$$

E running through the C-Azumaya algebras.

There is, for clean orders $A$ over a Dedekind domain a connection between Picent $(A)$ and the projective class group, which will be studied in a subsequent joint note of I. Reiner, S. Ullom and myself [FRU]. This leads, under certain conditions, to a longer exact sequence.

Proof of Corollary. The extra fact needed is that

$$
\operatorname{cent}\left(A \otimes_{C} E\right)=\operatorname{cent}(A)
$$

if $E$ is $C$-Azumaya. Given this, the Corollary is just a special case of the theorem.

To establish (1.3) consider a $C$-algebra $B$, and a $C$-Azumaya algebra $E$. Then cent $(B)$ embeds in cent $(B) \otimes_{C} E$ and as $E$ is flat this embeds in $B \otimes_{C} E$. In other words the composite map cent $(B) \rightarrow B \rightarrow B \otimes_{C} E$ yields an injection cent $(B) \rightarrow$ cent $\left(B \otimes_{C} E\right)$. Apply this first to $B=A$, with given $E$ and then to $B=A \otimes_{C} E$, and $E$ replaced by an $C$-Azumaya algebra $E_{1}$ so that $E \otimes_{C} E_{1}=$ $M_{n}(C)$ the ring of $n$ by $n$ matrices over $C$. We get injections $C=$ cent $(A) \rightarrow$ cent $\left(A \otimes_{C} E\right)$, cent $\left(A \otimes_{C} E\right) \rightarrow$ cent $\left(A \otimes_{C} M_{n}(C)\right)$ whose composite is clearly a bijection. Thus cent $(A) \rightarrow$ cent $\left(A \otimes_{C} E\right)$ is a bijection.

2. The group Picent. Let $\mathrm{Smalg}_{R}$ be the category of commutative $R$-algebras and their isomorphisms. Taking centres we get a functor

$$
\text { cent: } \operatorname{lug}_{R} \rightarrow \text { somalg }_{R} \text {. }
$$


This has the property that given an $R$-algebra $A$ and an automorphism $f$ of the $R$-algebra cent $(A)$ there is an $R$-algebra $B$ with cent $(B)=$ cent $(A)$ and an isomorphism $\bar{f}: A \rightarrow B$ of $R$-algebras so that $f=$ cent $(\bar{f})$. This determines uniquely the isomorphism class of $B$ as cent $(A)$-algebra, and we shall write $B=A^{f}$. We shall write $A u_{R}$ (cent $\left.(A) \mid \operatorname{llg}(A)\right)$ for the subgroup of $A u_{R}$ (cent $(A)$ ) of elements $f$ for which the isomorphism class of $A^{f}$ in $2 \mathrm{Mg}_{\text {cent }(A)}$ is that of $A$, and Aut $_{R}$ (cent $(A) \mid M-2 M g(A)$ ) for the subgroup of Aut $_{R}$ (cent $(A)$ ) of elements $f$, for which the isomorphism class of $A^{f}$ in $\Re_{-2} \mathrm{Mg}_{\text {cent }(A)}$ is that of $A$. Both these subgroups are stabilisers of the "class of $A$ " with respect to the action of Aut $_{R}$ (cent $\left.(A)\right)$ on the isomorphism classes "above cent $(A)$ " in $2 \mathbb{M g}_{\text {cent }(A)}$ and $M-2 \mathrm{Ug}_{\text {cent }(A)}$ respectively.

Theorem 2. (i) Given an invertible A-B-bimodule $M$ over $R$, there is a unique isomorphism

$$
\Phi(M)=f: \operatorname{cent}(B) \rightarrow \operatorname{cent}(A)
$$

of $R$-algebras, so that

$$
f(c) m=m c, \quad \forall m \in M, \forall c \in \operatorname{cent}(B) .
$$

This defines a functor

$$
\Phi: \stackrel{M}{M}-\operatorname{llg}_{R} \rightarrow \text { Somalg }_{R}
$$

so that the diagram

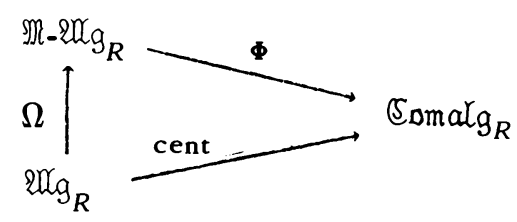

commutes.

(ii) The functors cent and $\Phi$ give rise to commutative diagrams with exact rows

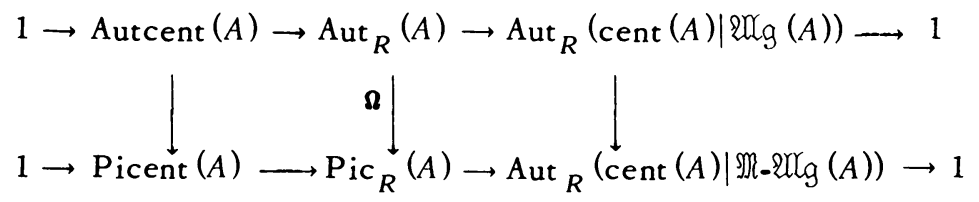

Remark. The exact sequence $1 \rightarrow \mathrm{Pic}_{C}(A) \rightarrow \mathrm{Pic}_{R}(A) \rightarrow \operatorname{Aut}_{R}(C)(C=$ cent $(A))$ is already given in [B1].

Proof of Theorem 2. (i) The map $f$ is the compositum of the isomorphism cent $(B) \cong \operatorname{End}\left({ }_{A} M_{B}\right)$ with the inverse of the isomorphism cent $(A) \cong \operatorname{End}\left({ }_{A} M_{B}\right)$. The remainder of (i) is now easy to verify. 
(ii) The only nontrivial part is the determination of the images of cent: Aut $_{R^{\prime}}(A) \rightarrow$ Aut $_{R}(\operatorname{cent}(A))$ and of $\Phi: \operatorname{Pic}_{R}(A) \rightarrow$ Aut $_{R}($ cent $(A))$. For the first it is clear that an automorphism $f$ of cent $(A)$ can be lifted to one of $A$ precisely when $A \cong A^{f}$, as cent $(A)$-algebras.

Next let $N=\Omega(\bar{f})$ be the invertible $A^{f}$-A-bimodule over $R$, corresponding under $\Omega$ to a lifting $\bar{f}: A \rightarrow A^{f}$ of $f \in$ Aut $_{R}$ (cent $\left.(A)\right)$. By (i), $\Phi(N)=f$. If $f \epsilon$ Aut $_{R}$ (cent $\left.(A) \mid M-20 \mathrm{lg}(A)\right)$ let $M$ be an invertible $A-A^{f}$-bimodule over cent $(A)$. Then $L=M \otimes_{A f} N$ is an invertible $A$-A-bimodule over $R$ with $\Phi(L)=f$. On the other hand, if $L$ is an invertible $A \cdot A$-bimodule over $R$ with $\Phi(L)=f$ and $N^{*}$ is the inverse bimodule of $N$, then $M=L \otimes_{A} N^{*}$ is an invertible $A-A^{f}$-bimodule over cent $(A)$. Thus indeed we get the required result on the image of $\Phi$.

Corollary $\mathbf{l}$ (cf. [Bl]). If $A$ is commutative, then the sequence

$$
1 \rightarrow \text { Picent }(A) \rightarrow \operatorname{Pic}_{R}(A) \rightarrow \operatorname{Aut}_{R}(A) \rightarrow 1
$$

is exact and splits.

For now Autcent $(A)=1$, and all the other three automorphism groups appearing in the diagram in (ii) coincide.

Corollary 2. Picent is a functor of $M-2 \mathrm{Mg}_{R}$, i.e. a Morita equivalence $B \sim A$ over $R$ yields an isomorpbism Picent $(B) \cong \operatorname{Picent}(A)$.

Proof. Immediate.

Corollary 3. Picent $(A)$ is a normal subgroup of $\mathrm{Pic}(A)$ (and of course Autcent $(A)$ a normal subgroup of Aut $(A))$.

Proof. Take $R=\mathbf{Z}$.

Next note that, if $\psi: T \rightarrow R$ is a homomorphism of commutative rings, then $\operatorname{Pic}_{R}(A) \subset \operatorname{Pic}_{T}(A), \operatorname{Aut}_{R}(A) \subset \operatorname{Aut}_{T}(A)$.

Corollary 4. If $\psi: T \rightarrow R$ is an epimorphism in the category of rings, then

$$
\operatorname{Pic}_{R}(A)=\operatorname{Pic}_{T}(A), \quad \operatorname{Aut}_{R}(A)=\operatorname{Aut}_{T}(A) .
$$

Note. This is of interest if $R$ is a residue ring or a ring of fractions of $T$.

Proof. Let $(X) \in \operatorname{Pic}_{T}(A)$. This means that if $j: R \rightarrow$ cent $(A)$ is the algebra structure map then $\Phi(X) \circ j \circ \psi=j \circ \psi$, hence $\Phi(X) \circ j=j$, i.e. $(X) \epsilon$ $\operatorname{Pic}_{R}(A)$. Similarly for $\mathrm{Aut}_{R}$.

Example. Suppose $A$ is separable over $R$, i.e. Azumaya over cent $(A)$. Then $A_{\text {ut }}$ (cent $(A) \mid M-2 M g A$ ) is just the subgroup of $A_{\text {ut }}{ }_{R}$ (cent $(A)$ ) which leaves the Brauer class of $A$ fixed. In many cases this group is easily determined, e.g. when we work over local or global fields or over algebraic integers. 
If cent $(A)$ is a field and $A$ is separable then $A$ ut ${ }_{R}(\operatorname{cent}(A) \mid \operatorname{latg} A$ )

$=\operatorname{Aut}_{R}(\operatorname{cent}(A) \operatorname{Mr}-2 \mathrm{Mg}(A))$. For if $f$ lies in the latter group and $A=M_{n}(D)$, the $n$ by $n$ matrix ring over the division algebra $D$, then $D \cong D^{f}$ over cent $(A)$, hence also $A \cong A^{f}$.

If $R$ is a field and $A$ semisimple, finite-dimensional over $R$, write

$$
A=\prod_{i} A_{i}, \quad \text { cent }(A)=\prod_{i} C_{i}
$$

where the $A_{i}$ are simple algebras, $C_{i}$ their centres. Let $f \in$ Aut $_{R}$ (cent $\left.(A)\right)$ with $C_{i}^{f}=C_{\phi(i)}$. Then a necessary and sufficient condition that $f \epsilon$ Aut $_{R}$ (cent $\left.(A) \mid M_{\text {- }} \operatorname{lng}(A)\right)$ is that for all $i$

$$
\operatorname{cl}\left(A_{i}^{f} / C_{\phi(i)}\right)=\operatorname{cl}\left(A_{\phi(i)} / C_{\phi(i)}\right)
$$

where $\operatorname{cl}\left(A_{j} / C_{j}\right)$ is the Brauer class of $A_{j}$ over $C_{j}$. Alternatively, if $f$ induces $f_{i}: C_{i} \cong C_{\phi(i)}$, we can write this as $\operatorname{cl}\left(A_{i} / C_{i}\right)^{f_{i}}=\operatorname{cl}\left(A_{\phi(i)} / C_{\phi(i)}\right)$.

We shall next use Theorem 2 to consider a product $A=\prod_{i \in I} A_{i}$ of $R$-algebras, $I$ being some not necessarily finite index set. Let $\Sigma_{R}\left(A_{i}, I\right)$ be the subgroup of the full permutation group of $I$ of permutations $\pi$, for which $A_{i}$ and $A_{\pi(i)}$ are Morita equivalent over $R$, for all $i$. Let $e_{i}$ be the idempotent of $\operatorname{cent}(A)$ with $A e_{i}=$ $e_{i} A=A_{i}$.

Theorem 3. (i) Let $\mathrm{Pic}_{R}^{*}(A)$ be the subgroup of $\mathrm{Pic}_{R}(A)$ of classes of bimodules $X$ with $X e_{i}=e_{i} X$ for all $i$. The maps $(X) \mapsto\left(X e_{i}\right)$ then define an injective bomomorphism

$$
\mu: \operatorname{Pic}_{R}^{*}(A) \rightarrow \prod_{i} \mathrm{Pic}_{R}\left(A_{i}\right)
$$

which is bijective if the index set I is finite.

(ii) If the $A_{i}$ are indecomposable R-algebras then with each $(X) \in \operatorname{Pic}_{R}(A)$ there is associated a unique $\pi \in \Sigma_{R}\left(A_{i}, I\right)$, so that

$$
x e_{i}=e_{\pi(i)} x, \quad \forall i \in I, \forall x \in X .
$$

The map $(X) \mapsto \pi$ is a bomomorphism $\operatorname{Pic}_{R}(A) \rightarrow \Sigma_{R}\left(A_{i}, I\right)$, which has kernel $\mathrm{Pic}_{R}^{*}(A)$, and which is surjective when I is finite. Thus, for finite I, we bave an exact sequence

$$
1 \rightarrow \prod_{i} \operatorname{Pic}_{R}\left(A_{i}\right) \rightarrow \mathrm{Pic}_{R}(A) \rightarrow \Sigma_{R}\left(A_{i}, I\right) \rightarrow 1
$$

Proof. If the $A_{i}$ are indecomposable, i.e. the $e_{i}$ are primitive idempotents of $\operatorname{cent}(A)$, then we have a homomorphism of Aut $(\operatorname{cent}(A))$ into the permutation group of $I, f$ being mapped into $\pi$, with $f\left(e_{i}\right)=e_{\pi(i)}$. The map $\operatorname{Pic}_{R}(A) \rightarrow \Sigma_{R}\left(A_{i}, I\right)$ is 
given by the composition of $\mathrm{Pic}_{R}(A) \rightarrow$ Aut (cent $(A) \mid \mathrm{M}-2 \mathrm{Mg}(A)$ ) with this map. It clearly has $\operatorname{Pic}_{R}^{*}(A)$ as kernel.

It is clear that in (i) $\mu$ is a well-defined homomorphism. To show that it is injective consider the natural homomorphisms

$$
l: M \rightarrow \prod_{i} e_{i} M, \quad r: N \rightarrow \prod_{i} N e_{i}
$$

for left $A$-modules $M$ and for right $A$-modules $N$. If $M=N$ is an $A$-A-bimodule with $e_{i} m=m e_{i}$ for all $m \in M$ and all $i$, the se two maps coincide and give a bimodule homomorphism. On the other hand if $M$ is finitely generated projective then $l$ is an isomorphism, this being so for $M={ }_{A} A$. Thus if $(X) \in \operatorname{Pic}_{R}^{*}(A)$ and $X e_{i} \cong A e_{i}$ as bimodules, then $X \cong A$ as bimodule. Thus $\mu$ is indeed injective.

Now suppose that $I$ is a finite set. If the $A_{i}$ are indecomposable, and $\pi \in \Sigma_{R}\left(A_{i}, I\right)$ choose $X_{i}$ as an invertible $A_{\pi(i)}-A_{i}$-bimodule over $R$. Then $X=$ $\Pi_{i} X_{i}$ maps onto $\pi$ under $\mathrm{Pic}_{R}(A) \rightarrow \Sigma_{R}\left(A_{i}, I\right)$. Next if $X_{i}$ are given invertible $A_{i}$-A $A_{i}$-bimodules over $R$, then $(X)=\left(\Pi_{i} X_{i}\right)$ maps onto $\Pi_{i}\left(X_{i}\right)$ under $\mu$.

Corollary 1. Picent $(A) \rightarrow \Pi_{i} \operatorname{Picent}\left(A_{i}\right)$ is injective, and for I finite, bijective.

Proof. Take $R=\operatorname{cent}(A)$. By Corollary 4 to Theorem $2, \operatorname{Pic}_{R}\left(A_{i}\right)=\operatorname{Picent}\left(A_{i}\right)$. Now apply Theorem 3.

Remark. Analogous results obviously hold for $A_{u t}$ and for Autcent.

3. Change of base ring. Throughout this section $\psi: R \rightarrow S$ is a homomorphism of commutative rings. We shall extend definitions and results of algebraic $K$-theory to the present situation, where the products involved are no longer necessarily commutative.

Let $A$ be a $T$-algebra, $R \rightarrow T$ a homomorphism of commutative rings. Throughout, the unadorned tensor product sign $\otimes$ will stand for $\otimes_{R} \cdot A \otimes S$ is then a $T \otimes S$-algebra. Define Out $_{T}(A)=$ Aut $_{T}(A) / \ln (A)$. We get homomorphisms

$$
\begin{aligned}
& \operatorname{Pic}_{T}(A) \rightarrow \mathrm{Pic}_{T \otimes S}(A \otimes S), \\
& \text { Aut }_{T}(A) \rightarrow \text { Aut }_{T \otimes S}(A \otimes S), \\
& \mathrm{Out}_{T}(A) \rightarrow \text { Out }_{T \otimes S}(A \otimes S),
\end{aligned}
$$

the first given by $(X) \mapsto(X \otimes S)$, the second by $f \mapsto f \otimes S$ and the third induced by the second.

We shall define certain relative groups. Consider pairs $(X, f), X$ an invertible $A$ - $A$-bimodule over $R$, and $f$ an isomorphism $X \otimes S \cong A \otimes S$ of $A \otimes S$ - $A \otimes S$-bimodules. An isomorphism $(X, f) \cong(Y, g)$ of such pairs is given by an isomorphism $b: X \cong Y$ of $A$-A-bimodules, so that the diagram 


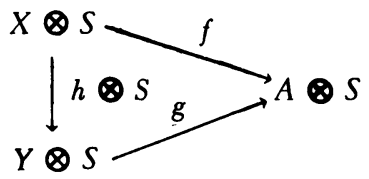

commutes. The product $\left(X_{1}, f_{1}\right) \otimes\left(X_{2}, f_{2}\right)$ is defined as $\left(X_{1} \otimes_{A} X_{2}, f_{1} \cdot f_{2}\right)$ where $f_{1} \cdot f_{2}$ is the compositum

$\left(X_{1} \otimes_{A} X_{2}\right) \otimes S \cong\left(X_{1} \otimes S\right) \otimes_{A \otimes S}\left(X_{2} \otimes S\right) \stackrel{f_{1}, f_{2}}{\longrightarrow}(A \otimes S) \otimes_{A \otimes S}(A \otimes S)=A \otimes S$.

The isomorphism classes of the $(X, f)$ form a group $\mathrm{Pic}_{\psi}(A)$ with respect to this product, those with $X$ being "over $T$ " a subgroup $\operatorname{Pic}_{\psi, T}(A)$. The map $((X, f)) \mapsto$ $(X)$ is a homomorphism

$$
\mathrm{Pic}_{\psi}(A) \rightarrow \mathrm{Pic}_{R}(A) .
$$

On the other hand, if we associate with $u \in U(\operatorname{cent}(A \otimes S))(U()$ is always the group of units) the pair $\left(A, g_{u}\right)$, where $g_{u}(a \otimes s)=u(a \otimes s)$, we get a homomorphism

$$
U(\text { cent }(A \otimes S)) \rightarrow \mathrm{Pic}_{\psi}(A) \text {. }
$$

Let now $M$ be an invertible $A-B$-bimodule over $R$, with inverse module $M^{*}$. With every pair $(X, f)$, as above, we associate the pair $\left(M^{*} \otimes_{A} X \otimes_{A} M, f^{\prime}\right)$, where $f^{\prime}$ is the compositum

$\left(M^{*} \otimes_{A} X \otimes_{A} M\right) \otimes S \cong M^{*} \otimes_{A}(X \otimes S) \otimes_{A} M \stackrel{\text { from } f}{\simeq} M^{*} \otimes_{A}(A \otimes S) \bigotimes_{A} M=B \otimes S$, the last isomorphism being that defining $M^{*}$ as inverse of $M$. This yields an isomorphism

$$
\operatorname{Pic}_{\psi}(A) \cong \operatorname{Pic}_{\psi}(B)
$$

Next let $\operatorname{Aut}_{\psi}(A)$ be the fibre product (pull back) with respect to the diagram

$$
\operatorname{Aut}_{R}(A) \longrightarrow \operatorname{Aut}_{S}(A \otimes S) .
$$

This is the group of pairs $(f, u), f \in \operatorname{Aut}_{R}(A), u \in U(A \otimes S)$ for which $u(a \otimes 1) u^{-1}=$ $f(a) \otimes 1, \forall a \in A$. Inserting the maps $U(A) \rightarrow$ Aut $_{R}(A), U(A) \rightarrow U(A \otimes S)$ in the appropriate places, the above diagram would be commutative, and hence we get a homomorphism 


$$
\iota_{\psi, A}: U(A) \rightarrow \mathrm{Aut}_{\psi}(A)
$$

which takes $v$ into the pair $\left(s_{v}, v \otimes 1\right)$, where $s_{v}$ is the inner automorphism induced by $v$. We define

$$
\operatorname{Out}_{\psi}(A)=\operatorname{Aut}_{\psi}(A) / \operatorname{Im} l_{\psi, A} \cdot
$$

Restricting to automorphisms in $\operatorname{Aut}_{T}(A)$, we get subgroups $\operatorname{Aut}_{\psi, T}(A)$, and Out $_{\psi, T}(A)$ of the above groups. Associating with $u \in U(\operatorname{cent}(A \otimes S))$ the pair $(1, u)$, we get homomorphisms

$$
\begin{aligned}
& U(\text { cent }(A \otimes S)) \rightarrow \text { Aut }_{\psi}(A), \\
& U(\text { cent }(A \otimes S)) \rightarrow \mathrm{Out}_{\psi}(A) .
\end{aligned}
$$

Next the map $(f, u) \mapsto f$ yields homomorphisms

$$
\begin{aligned}
& \operatorname{Aut}_{\psi}(A) \rightarrow \operatorname{Aut}_{R}(A), \\
& \mathrm{Out}_{\psi}(A) \rightarrow \mathrm{Out}_{R}(A) .
\end{aligned}
$$

Next if $(f, u) \in \operatorname{Aut}_{\psi}(A)$, then $g\left(a_{f} \otimes s\right)=(a \otimes s) u$ defines an isomorphism $g: A_{f} \otimes S \cong A \otimes S$ of $A \otimes S-A \otimes S$-bimodules. If $(f, u) \in \operatorname{Im} l_{\psi, A}$ then the pair $\left(A_{f}, g\right)$ is isomorphic to $(A, 1)$. Thus the map $(f, u) \mapsto\left(\left(A_{f}, g\right)\right)$ yields homomorphisms

$$
\begin{aligned}
& \operatorname{Aut}_{\psi}(A) \rightarrow \operatorname{Pic}_{\psi}(A), \\
& \text { Out }_{\psi}(A) \rightarrow \operatorname{Pic}_{\psi}(A) .
\end{aligned}
$$

Now suppose that $A \rightarrow A \otimes S$ is injective. This is a property preserved by Morita equivalence. View $A$ as embedded in $A \otimes S$. We define the product $V W$ of $A-A$-subbimodules $V$ and $W$ of $A \otimes S$ as the module of sums $\Sigma_{v_{i}} w_{i}, v_{i} \in V$, $w_{i} \in W$. Call $V$ an invertible fractional ideal of $A$ in $A \otimes S$ if, for some $W, V W=$ $W V=A$. These form a group.under the product we have just defined, to be denoted by $I_{\psi}(A)$; or just $I(A)$. The normaliser $N_{\psi}(A)$ or $N(A)$ is the subgroup of $U(A \otimes S)$ of elements $u$ with $u A u^{-1}=A$.

Now we can at last state our theorem, where the new maps are those explicitly defined above, or are obvious.

Theorem 4. Let $\psi: R \rightarrow S$ be a bomomorphism of commutative rings, $R \rightarrow T$ a bomomorphism of commutative rings and A a T-algebra.

(i) We have a commutative diagram with exact rows and columns. 


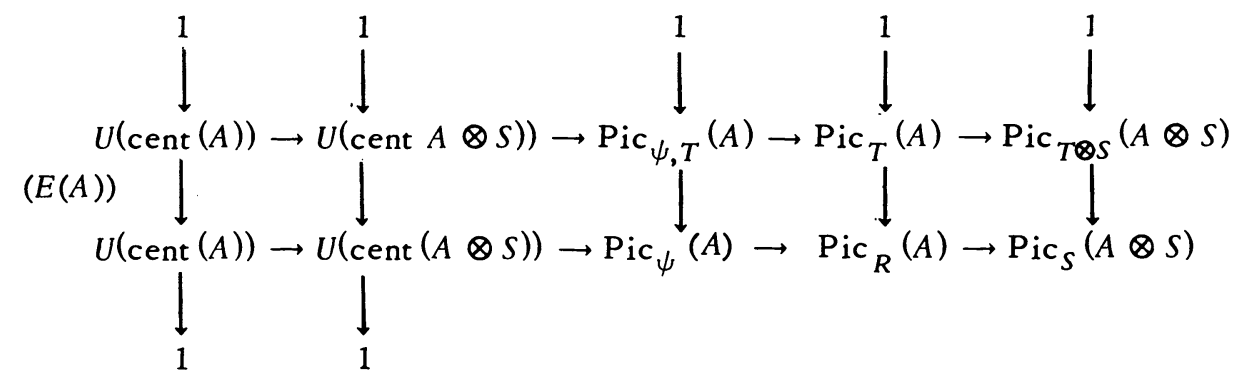

$A$ Morita equivalence $A \sim B$ over $T$ yields an isomorphism $E(A) \cong E(B)$ of diagrams.

(ii) We bave a commutative diagram

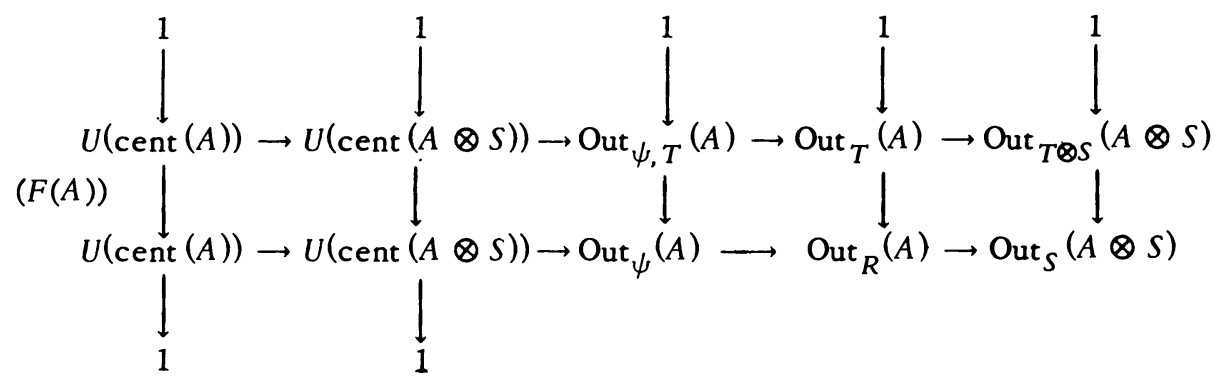

with exact rows and columns.

The maps $\mathrm{Out}_{\psi}(A) \rightarrow \mathrm{Pic}_{\psi}(A)$ and $\mathrm{Out}_{\psi, T}(A) \rightarrow \mathrm{Pic}_{\psi, T}(A)$ are injective and yield, together with the maps Out $\rightarrow$ Pic induced by $\Omega$ a bomomorphism $(F(A)) \rightarrow$ $(E(A))$ of diagrams.

(iii) Suppose now that $A \subset A \otimes S$. Then every invertible fractional ideal of $A$ in $A \otimes S$ is an invertible $A-A$-bimodule over $R$. Associating with each such fractional ideal $V$ the pair $\left(V, f_{V}\right)$, where $f_{V}: V \otimes S \rightarrow A \otimes S$ is induced by $V \hookrightarrow A \otimes S$, we get an isomorphism $I_{\psi}(A) \cong \operatorname{Pic}_{\psi}(A)$, and a commutative diagram.

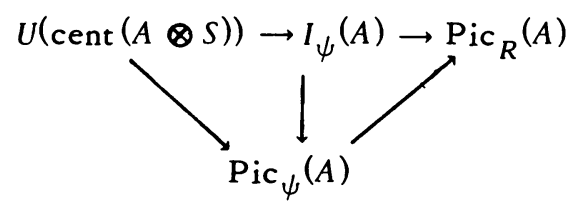

Here the maps of the top row are $u \mapsto u A$ and $V \mapsto(V)$ respectively.

The map $u \mapsto(f, u), u \in N_{\psi}(A)$, f the automorphism of $A$ induced by $u$, is an isomorpbism $N_{\psi}(A) \cong \mathrm{Aut}_{\psi}(A)$, and giving rise to an isomorpbism $N_{\psi}(A) / U(A) \cong$ Out $_{\psi}(A)$. The map $u \mapsto u A=A u, u \in N_{\psi}(A)$, yields a homomorphism, with kernel $U(A), N_{\psi}(A) \rightarrow I_{\psi}(A)$, so that the diagram 


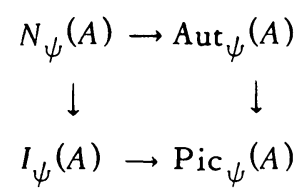

commutes.

Proof. The main work leading to the theorem has already been done by defining the appropriate maps. The verification of the exactness of the rows of $(E(A))$ and $(F(A))$ is now purely routine, following the standard procedures of $K$ theory. The commutativity of the diagrams, the Morita invariance of $(E(A))$ and the homomorphism $(F(A)) \rightarrow(E(A))$ are all obvious. The exactness of the columns in both diagrams is already known to us, except for the middle column where it is a consequence of the others or of the definition. The same type of reasoning yields the injectivity of $\mathrm{Out}_{\psi} \rightarrow \mathrm{Pic}_{\psi}$.

For (iii) we have to do a bit more work. If $V, W \in I_{\psi}(A)$, multiplication in $A \otimes S$ yields a homomorphism $q: V \otimes_{A} W \rightarrow V W$ of $A$ - $A$-bimodules, clearly surjective. Let $\Sigma v_{i} \otimes w_{i} \in \operatorname{Ker} q$. Suppose $V V^{\prime}=V^{\prime} V=A$, with $\Sigma x_{k} y_{k}=1$, $x_{k} \in V, y_{k} \in V^{\prime}$. Then

$$
\sum_{i} v_{i} \otimes w_{i}=\sum_{i, k} x_{k} y_{k} v_{i} \otimes w_{i}=\sum_{i, k} x_{k} \otimes y_{k} v_{i} w_{i}=0
$$

Thus $q$ is an isomorphism. In particular we now get isomorphisms $V \otimes_{A} V^{\prime} \cong A$, $V^{\prime} \otimes_{A} V \cong A$, which by the associative law for multiplication in $A \otimes S$ satisfy condition (II) in $\$ 1$. Thus $(V) \in \operatorname{Pic}_{R}(A)$, the fact that $V$ is "over $R$ " being trivial. Provided we can show that $f_{V}$, as defined in the theorem, is an isomorphism we thus have a homomorphism $w: I_{\psi}(A) \rightarrow \mathrm{Pic}_{\psi}(A)$.

Identify $V \otimes S=V \otimes_{A}(A \otimes S)$. Then $f_{V}(v \otimes b)=v b$, for $v \in V, b \in A \otimes S$. As $V$ is invertible, $f_{V}$ is surjective. If $\Sigma v_{i} \otimes_{A} b_{i} \in \operatorname{Ker} f_{V}, v_{i} \in V, b_{i} \in A \otimes S$, then with $x_{k}, y_{k}$ as above we have

$$
\sum_{i} v_{i} \otimes b_{i}=\sum_{i, k} x_{k} y_{k} v_{i} \otimes b_{i}=\sum_{i, k} x_{k} \otimes y_{k} v_{i} b_{i}=0
$$

Thus $f_{V}$ is an isomorphism.

Next we shall show that $w$ is an isomorphism, by displaying its inverse map. Let $((X, f)) \in \operatorname{Pic}_{\psi}(A)$, with inverse $((Y, g))$, and write $f(X)$ for the image of the composite map $X \rightarrow X \otimes S \stackrel{f}{\longrightarrow} A \otimes S$. Then $f(X)$ solely depends on the class of $(X, f)$, and $f(X) g(Y)=g(Y) f(X)=A$. Thus $((X, f)) \mapsto f(X)$ is a well-defined map $w^{\prime}: \operatorname{Pic}_{\psi}(A) \rightarrow I_{\psi}(A)$ with $w^{\prime} \circ w=1$. To show that also $w \circ w^{\prime}=1$, we have to prove that $X \cong f(X)$ as bimodules, i.e. that $X \rightarrow X \otimes S$ is injective. In fact $U \rightarrow U \otimes S$ is injective for every finitely generated, projective (say left) $A$-module $U$, this being so for $U=A$. 
The assertions with respect to $N_{\psi}(A)$ are straightforward to check.

For $T=\operatorname{cent}(A)$ write Outcent $(A)=\operatorname{Out}_{T}(A), \operatorname{Picent}_{\psi}(A)=\operatorname{Pic}_{\psi, T}(A)$,

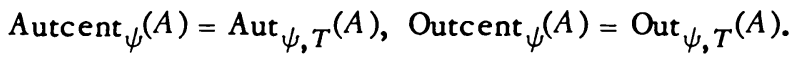

Corollary 1. (i) Suppose that cent $(A) \otimes S \rightarrow \operatorname{cent}(A \otimes S)$ is surjective. Then we have exact sequences

$U($ cent $(A)) \rightarrow U($ cent $(A \otimes S)) \rightarrow$ Picent $_{\psi}(A) \rightarrow$ Picent $(A) \rightarrow$ Picent $(A \otimes S)$, $U($ cent $(A)) \rightarrow U($ cent $(A \otimes S)) \rightarrow$ Outcent $_{\psi}(A) \rightarrow$ Outcent $(A) \rightarrow$ Outcent $(A \otimes S)$.

(ii) Suppose moreover that $\operatorname{cent}(A) \rightarrow \operatorname{cent}(A) \otimes S$ is injective. Then

$$
\begin{aligned}
& \operatorname{Picent}_{\psi}(A)=\operatorname{Pic}_{\psi}(A), \\
& \text { Autcent }_{\psi}(A)=\text { Aut }_{\psi}(A), \\
& \text { Outcent }_{\psi}(A)=\operatorname{Out}_{\psi}(A) .
\end{aligned}
$$

Proof. (i) From the theorem and Corollary 4 to Theorem 2.

(ii) By Theorem 2 we have a commutative diagram

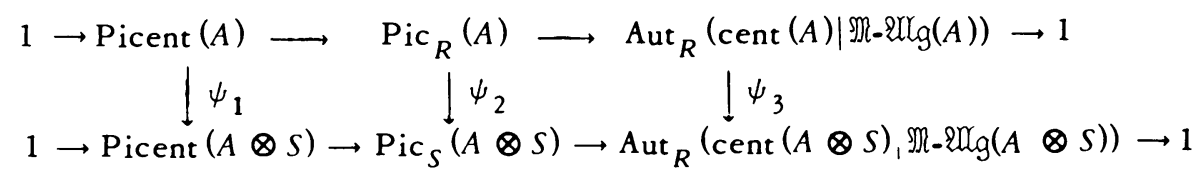

with exact rows. The hypothesis implies that $\psi_{3}$ is injective, hence $\operatorname{Ker} \psi_{1} \cong$ $\operatorname{Ker} \psi_{2}$, and thus by Theorem 4 (diagram $(E(A))$ implies that the subgroup Picent $\psi^{(A)}$ actually is the whole of $\mathrm{Pic}_{\psi}(A)$. A similar argument yields the result for Outcent, and that for Autcent is an immediate consequence.

Condition (i) holds if $S$ is flat over $R$ and $A$ is finitely generated as $R$-module (see e.g. Lemma $A$ in $\$ 4$ ).

Next we get

Corollary 2. Let $R$ be an integral domain with quotient field $K$ and let $A$ be a finitely generated, torsion free $R$-module and an $R$-algebra so that $A \otimes K$ is semisimple. Let $I(A)$ be the group of invertible fractional ideals of $A$ in $A \otimes K$ and $N(A)$ the normaliser of $A$ in $A \otimes K$. Then the following sequences are exact:

(a) $1 \rightarrow U(\operatorname{cent}(A \otimes K)) / U(\operatorname{cent}(A)) \rightarrow N(A) / U(A) \rightarrow \operatorname{Outcent}(A) \rightarrow 1$,

(b) $1 \rightarrow U(\operatorname{cent}(A \otimes K)) \rightarrow \mathrm{N}(A) \rightarrow$ Autcent $(A) \rightarrow 1$,

(c) $U(\operatorname{cent}(A \otimes K)) \rightarrow I(A) \rightarrow \operatorname{Picent}(A) \rightarrow 1$,

(d) $1 \rightarrow \operatorname{Picent}(A) \rightarrow \operatorname{Pic}_{R}(A) \rightarrow \operatorname{Pic}_{K}(A \otimes K)$,

(e) $1 \rightarrow U(A) / U(\operatorname{cent}(A)) \stackrel{R}{\rightarrow} N(A) / U(\operatorname{cent}(A \otimes K)) \stackrel{\Omega^{\prime}}{\rightarrow} \operatorname{Picent}(A)$

and the cosets in $\operatorname{Picent}(A) / \operatorname{Im} \Omega^{\prime}$ are the fibres of $\operatorname{Picent}(A) \rightarrow \operatorname{Proj}(A)$.

Remark. (c) and (d) yield new characterisations of Picent. In particular (c) 
shows that Picent is a generalisation of the classical ideal class group. The group $I(A)$ for certain orders $A$ has been considered classically (see [DE]) and since.

Proof. That Picent $(A \otimes K)=1$ is well known. In any case the proof of this reduces by the Corollary to Theorem 3 to the case when $A \otimes K$ is simple, i.e. is Azumaya over a field $L$. As remarked earlier (after (1.2)) then Picent $(A \otimes K)=$ Picent $(L)$, and the latter $=1$ as $L$ is a field. By Corollary 1 (i) and (ii) (the conditions for which certainly hold here), we get a surjection $\operatorname{Pic}_{\psi}(A) \rightarrow \operatorname{Picent}(A)$, and by Theorem 4 (commutativity of $E(A)$ ), the map $\mathrm{Pic}_{\psi}(A) \rightarrow \mathrm{Pic}_{R}(A)$ factors through the injection $\mathrm{Picent}(A) \rightarrow \mathrm{Pic}_{R}(A)$. By Theorem 4 (i) we now conclude that (d) is exact, hence (c) is exact.

Analogously we get a surjection $\mathrm{Out}_{\psi}(A) \rightarrow \operatorname{Outcent}(A)$ with kernel $U(\operatorname{cent}(A \otimes K)) / U(\operatorname{cent}(A))$, yielding a surjection $N(A) \rightarrow$ Autcent $(A)$ with the obvious kernel $U(\operatorname{cent}(A \otimes K))$. Thus we have the exactness of (a), (b). That of (e) and the characterisation of $\operatorname{Picent}(A) / \operatorname{Im} \Omega^{\prime}$ follows now from the corollary to Theorem 1 .

Corollary 3. With $R$ and $A$ as in Corollary 2, suppose moreover that $R$ is a discrete valuation ring and let $\bar{R}$ be its completion. Then

$$
\begin{array}{cc}
I(A) \cong I(A \otimes \bar{R}), & \text { Picent }(A) \cong \operatorname{Picent}(A \otimes \bar{R}), \\
N(A) / U(A) \cong N(A \otimes \bar{R}) / U(A \otimes \bar{R}), & \text { Outcent }(A) \cong \text { Outcent }(A \otimes \bar{R}) .
\end{array}
$$

(Here $I$ and $N$ are defined via the maps $\psi: R \rightarrow K$, and $\bar{\psi}: \bar{R} \rightarrow \bar{R} \otimes K=\bar{K}$.)

Proof. Using the obvious natural properties of the diagrams of Theorem 4 for commutative diagrams

$$
\underset{R^{\prime} \stackrel{\psi^{\prime}}{\rightarrow} S^{\prime}}{\stackrel{\psi}{\rightarrow} S}
$$

we have a commutative diagram with exact rows (and with $\bar{A}=A \otimes \bar{R}$ )

$$
\begin{gathered}
1 \rightarrow U(\text { cent }(A)) \rightarrow U(\text { cent }(A \otimes K)) \rightarrow I(A) \rightarrow \text { Picent }(A) \rightarrow 1 \\
\downarrow \\
1 \rightarrow U(\text { cent }(\bar{A})) \rightarrow U\left(\text { cent }\left(\bar{A} \otimes_{\bar{R}} \bar{K}\right) \rightarrow I(\bar{A}) \rightarrow \text { Picent }(\bar{A}) \rightarrow 1\right.
\end{gathered}
$$

$b$ is an isomorphism, induced by the bijections between $R$-lattices in $A \otimes K$ and $\bar{R}$-lattices in $\bar{A} \otimes_{\bar{R}} \bar{K}$. Thus $k$ is surjective. As $U(\operatorname{cent}(\bar{A}))$ isopen in $U\left(\operatorname{cent}\left(\bar{A} \otimes_{\bar{R}} \bar{K}\right)\right.$ ), and $U(\operatorname{cent}(A \otimes K))$ is dense in it, the product of these two subgroups is the whole of $U\left(\operatorname{cent}\left(\bar{A} \otimes_{\bar{R}} \bar{K}\right)\right.$. Now a bit of diagram chasing shows that $k$ is injective.

We have a commutative diagram 


$$
\begin{array}{cc}
N(A) / U(A) & \rightarrow I(A) \\
\downarrow & \stackrel{\downarrow}{\downarrow}(\bar{A}) / U(\bar{A}) \rightarrow I(\bar{A})
\end{array}
$$

with injective rows and right hand column. Thus $N(A) / U(A) \rightarrow N(\bar{A}) / U(\bar{A})$ is injective. As $N(A)$ is dense in $N(\bar{A})$ and $U(\bar{A})$ open, that map is also surjective. For the last isomorphism one now uses a diagram as above with $I$ replaced by $N / U$ and Picent by Outcent.

4. The localisation sequence and central embedding.

Notation. If $p$ is a prime ideal of the commutative ring $R$, write $M_{p}$ for the localisation at $p$ of an $R$-module $M$. In particular $R_{p}$ is the local ring of $R$ at $p$ and $M_{p} \cong M \dot{\otimes}_{R} R_{p}$.

The localisation maps $\mathrm{Pic}_{R}(A) \rightarrow \mathrm{Pic}_{R_{\mathfrak{p}}}\left(A_{\mathfrak{p}}\right)$ clearly commute with the isomorphisms induced by Morita equivalence, and with the tensor product $A_{1} \otimes_{R} A_{2}$ of algebras. The latter applies also to the localisation maps $\operatorname{Aut}_{R}(A) \rightarrow \operatorname{Aut}_{R_{p}}\left(A_{p}\right)$.

We have also to consider the homomorphism I': Picent(cent $(A)) \rightarrow \operatorname{Picent}(A)$, given by $(M) \mapsto(M \otimes \operatorname{cent}(A) A)$. This again commutes with Morita equivalence over cent $(A)$, and if $E$ is a cent $(A)$-Azumaya algebra then the diagram

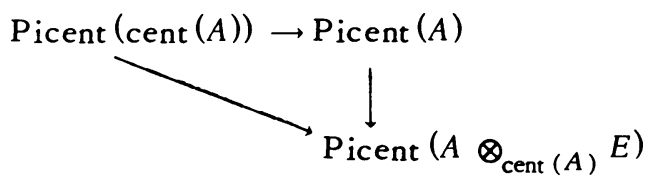

will commute.

One more notation: If $Y$ is an $A$ - $A$-bimodule then

$$
Y^{A}=[y \in Y \mid y a=a y \quad \forall a \in A] .
$$

$Y^{A}$ is a cent $(A)$-module.

Theorem 5. Let $A$ be an R-algebra.

(i) The map $\mathrm{T}: \mathrm{Picent}(\mathrm{cent}(A)) \rightarrow \mathrm{Picent}(A)$ is injective and $\operatorname{Im} \mathrm{T}$ is central in Picent $(A)$.

(ii) $\operatorname{Ker}\left[\mathrm{Pic}_{R}(A) \rightarrow \Pi_{\mathfrak{p}} \operatorname{Pic}_{R}\left(A_{\mathfrak{p}}\right)\right] \subset \operatorname{Picent}(A)$.

(iii) If for every maximal ideal $\mathrm{m}$ of $R$, cent $(A)_{\mathfrak{m}}=($ cent $(A)) \otimes_{R} R_{\mathfrak{m}}$ is semilocal (i.e. bas only a finite number of maximal ideals) then

$$
\operatorname{Im} \mathrm{T} \subset \operatorname{Ker}\left[\text { Picent }(A) \rightarrow \prod_{p} \mathrm{Pic}_{R}\left(A_{p}\right)\right] .
$$

(iv) If, for every invertible A-A-bimodule $Y, Y^{A}$ is finitely generated over cent $(A)$ and, for all maximal ideals $m$ of $R$, the map $\left(Y^{A}\right)_{\mathfrak{m}} \rightarrow Y_{\mathfrak{m}}^{A_{\mathfrak{m}}}$ induced by 
$Y^{A} \rightarrow Y$ is bijective, then

$$
\operatorname{Ker}\left[\text { Picent }(A) \rightarrow \prod_{p} \operatorname{Pic}_{R}\left(A_{p}\right)\right] \subset \operatorname{Im} \mathrm{T} \text {. }
$$

(v) If cent $(A)_{\mathfrak{m}}$ is semilocal for every maximal ideal of $R$, cent $(A)$ is Noetberian and $A$ is finitely generated over cent $(A)$, then the sequence

(a) $1 \rightarrow \operatorname{Picent}($ cent $(A)) \rightarrow \operatorname{Pic}_{S}(A) \rightarrow \Pi_{\mathfrak{p}} \operatorname{Pic}_{S}\left(A_{p}\right)$

is exact for $S=R$, or more generally whenever $A$ is an $S$-algebra and $R \rightarrow S a$ bomomorphism of commutative rings. Moreover we have an exact sequence

(b) $1 \rightarrow \operatorname{Picent}(\operatorname{cent}(A)) \rightarrow \operatorname{Picent}(A) \rightarrow \Pi_{\mathfrak{p}} \operatorname{Picent}\left(A_{\mathfrak{p}}\right)$.

Througbout $\Pi_{p}$ runs over a subset of $\operatorname{spec}(R)$ containing all maximal ideals.

Remarks. (1) Recall that $\operatorname{Pic}_{s}\left(A_{\mathfrak{p}}\right)=\operatorname{Pic}_{s_{\mathfrak{p}}}\left(A_{\mathfrak{p}}\right)$, by Corollary 4 Theorem 2 .

(2) The hypothesis in (v) holds e.g. if $R$ is Noetherian and $A$ is a finitely generated $R$-module.

The proof depends on two lemmas.

Lemma A. Let $A$ be an algebra over a commutative ring $S$, finitely generated as S-module and let $T$ be a commutative, flat $S$-algebra. Then for any A-A-bimodule $Y$,

$$
Y^{A} \otimes_{S} T \cong\left(Y \otimes_{S} T\right)^{A \otimes T}
$$

Proof. Let $A^{e}=A \otimes_{S} A^{\text {op }}, A^{\text {op }}$ the opposite algebra, and consider the exact sequence

$$
0 \rightarrow \operatorname{Ker} f \rightarrow A^{e} \stackrel{f}{\rightarrow} A \rightarrow 0
$$

$f\left(a \otimes b^{\mathrm{op}}\right)=a b$. If $\left\{a_{i}\right\}$ is a set of generators of $A$ as $S$-module, then $\left\{a_{i} \otimes 1-1 \otimes a_{i}\right\}$ is a set of generators of $\operatorname{Ker} f$ as $A^{e}$-module. Thus $A$ is a finitely presented $A^{e}$-module, and hence by a standard argument,

$$
\operatorname{Hom}_{A} e^{(A, Y) \otimes_{S} T \cong \operatorname{Hom}_{A} e_{\otimes_{S}} T}\left(A \otimes_{S} T, Y \otimes_{S} T\right),
$$

which is exactly what was required.

Next we consider the natural homomorphism $w_{Y}: Y^{A} \otimes_{\operatorname{cent}(A)} A \rightarrow Y$, $\left(w_{Y}(y \otimes a)=y a\right)$ for $A$-A-bimodules $Y$.

Lemma B. If $(X) \in$ Picent(cent $(A))$ then $X \cong\left(X \otimes_{\text {cent }(A)} A\right)^{A}$.

Let $(Y) \in \operatorname{Pic}(A)$. Then $(Y) \in \operatorname{Im} \mathrm{T}$ if and only if firstly $\left(Y^{A}\right) \epsilon$ Picent(cent $(A)$ ), and secondly $w_{Y}$ is an isomorphism.

Proof. The natural map for cent $(A)$-modules

$$
X \rightarrow\left(X \otimes_{\operatorname{cen}(A)} A\right)^{A} \quad(x \mapsto x \otimes 1)
$$


is an isomorphism for $X=$ cent $(A)$, hence for all finitely generated, projective $X$.

This result implies that whenever $(Y) \in \operatorname{Im} \mathrm{T}$, then the stated conditions are satisfied. The converse is immediate.

Proof of Theorem 5. (i) The injectivity of $\mathrm{T}$ follows from the first part of Lemma $\mathrm{B}$. If $X$ is a cent $(A)$-module, $Y$ an $A-A$-bimodule over cent $(A)$, then the interchange $x \otimes y \mapsto y \otimes x$ is an isomorphism $X \otimes_{\text {cent }(A)} Y \cong Y \otimes_{\text {cent }(A)} X$. As $\left(X \otimes_{\text {cent }(A)} A\right) \otimes_{A} Y \cong X \otimes_{\text {cent }(A)} Y$, and similarly on the other side, Im $\mathrm{T}$ is indeed central in $\operatorname{Picent}(A)$.

In the remainder of the proof we may suppose that $\Pi_{\mathfrak{p}}=\Pi_{m}$ runs precisely over the maximal ideals $m$ of $R$. For, the kernel of the map into the product of the localisations is always the same provided that all maximal ideals occur.

(ii) Let $(X) \in \operatorname{Ker}\left[\mathrm{Pic}_{R}(A) \rightarrow \Pi_{\mathrm{m}} \mathrm{Pic}_{R}\left(A_{\mathrm{m}}\right)\right]$. With $c \in$ cent $(A)$ associate the endomorphism $f$ of $X$, where $f(x)=c x-x c$. As cent $(A)_{m}$ embeds in cent $\left(A_{m}\right)$, and as $X_{\mathrm{m}} \cong A_{\mathrm{m}}$ as bimodules, $f_{\mathrm{m}}=0$. This being so for all $\mathrm{m}, f=0$. Thus indeed $(X) \in \operatorname{Picent}(A)$.

(iii) Picent (cent $(A)) \rightarrow \operatorname{Picent}(A) \rightarrow \operatorname{Pic}_{R}\left(A_{\mathrm{m}}\right)$ factorises through Picent (cent $\left.(A)_{\mathfrak{m}}\right)$. But this group vanishes, cent $(A)_{\mathfrak{m}}$ being semilocal.

(iv) Suppose that, for all $\mathrm{m}, Y_{\mathrm{m}} \cong A_{\mathrm{m}}$ (isomorphism of bimodules). Then $Y_{\mathrm{m}}^{A_{\mathrm{m}}} \cong \operatorname{cent}\left(A_{\mathrm{m}}\right)$. But $Y_{\mathrm{m}}^{A_{\mathrm{m}}} \cong\left(Y^{A}\right)_{\mathrm{m}}$, in particular $\operatorname{cent}\left(A_{\mathrm{m}}\right) \cong \operatorname{cent}(A)_{\mathrm{m}}$. Thus for all maximal ideals $\mathrm{m}$ of $R,\left(Y^{A}\right)_{\mathrm{m}} \cong$ cent $(A)_{\mathrm{m}}$. This then holds for all prime ideals of $R$, hence for all prime ideals of cent $(A)$. As $Y^{A}$ is finitely generated over $\operatorname{cent}(A)$ it follows that $Y^{A}$ is a rank one projective over $\operatorname{cent}(A)$, so $\left(Y^{A}\right) \epsilon$ Picent $(\operatorname{cent}(A))$.

The map $\left(w_{Y}\right)_{m}$ factorises as

$$
\left(Y^{A} \otimes_{\operatorname{cent}\left(A_{\mathrm{m}}\right)} A\right)_{\mathrm{m}} \stackrel{f}{\cong}\left(Y^{A}\right)_{\mathrm{m}} \otimes_{\operatorname{cent}(A)_{\mathrm{m}}} A_{\mathrm{m}} \stackrel{g}{\rightarrow} Y_{\mathrm{m}}^{A_{\mathrm{m}}} \otimes_{\text {cent }(A)_{\mathrm{m}}} A_{m} \stackrel{{ }^{w} Y_{\mathrm{t.} .}}{\longrightarrow} Y_{\mathrm{m}},
$$

where $f$ is the natural isomorphism of the tensor product on ring extension, $g$ is an isomorphism, as $\left(Y^{A}\right)_{\mathfrak{m}} \cong Y_{\mathfrak{m}}^{A_{\mathfrak{m}}}$ and in particular cent $(A)_{\mathfrak{m}} \cong \operatorname{cent}\left(A_{\mathrm{m}}\right)$, and $w_{Y_{\mathrm{m}}}$ is an isomorphism, as $Y_{\mathfrak{m}} \cong A_{\mathrm{m}}$. Thus $\left(w_{Y}\right)_{\mathfrak{m}}$ is an isomorphism for all maximal ideals $m$ of $R$. Hence $w_{Y}$ is an isomorphism. By Lemma $\mathrm{B},(Y) \in \operatorname{Im} \mathrm{T}$.

(v) We assume the hypotheses of (v). Apply Lemma $A$ to $S=\operatorname{cent}(A), T=$ $\operatorname{cent}(A)_{\mathrm{m}}$, for $\mathrm{m}$ a maximal ideal of $R$, and we deduce that $\left(Y^{A}\right)_{\mathrm{m}} \cong Y_{\mathrm{m}}^{A_{\mathrm{m}}}$ as required under (iv). Moreover every finitely generated projective left $A$-module $Y$ is finitely generated over cent $(A)$, this being so for $Y=A$. As cent $(A)$ is Noetherian, it follows for any invertible $A$ - $A$-bimodule $Y$ that the cent $(A)$-submodule $Y^{A}$ is finitely presented. Thus the hypothesis of both (iii) and (iv) hold, and hence the sequence

$$
\text { (c) } 1 \rightarrow \operatorname{Picent}(\operatorname{cent}(A)) \stackrel{\mathrm{T}}{\rightarrow} \operatorname{Picent}(A) \stackrel{\lambda}{\rightarrow} \Pi_{\mathrm{m}} \mathrm{Pic}_{R}\left(A_{\mathrm{m}}\right) \text { is exact. }
$$


The conclusion in (ii) clearly implies an analogous statement with $R$ replaced by $S(S$ as under $(v))$. The exactness of (c) thus implies the exactness of the sequence $(a)$ in $(v)$. We have moreover seen that the hypotheses of $(v)$ imply those of (iv) and in particular that cent $(A)_{\mathfrak{m}} \cong \operatorname{cent}\left(A_{\mathfrak{m}}\right.$ ) for all $\mathrm{m}$. But then the exactness of (b) follows from that of (a) for $S=\operatorname{cent}(A)$, as $\operatorname{Pic}_{S}\left(A_{m}\right)=\operatorname{Pic}_{S_{\mathrm{m}}}\left(A_{\mathrm{m}}\right)$ by Corollary 4 to Theorem 2 .

5. Localisation for orders. An $R$-algebra $A$ is an order over $R$, if $A$ is finitely generated and projective as an $R$-module.

Theorem 6. Let $R$ be a Dedekind domain with quotient field $K$, and $A$ an order over $R$, so that $A \otimes K$ is a semisimple algebra. Then

(i) $I(A) \cong \amalg_{\mathrm{m}} I\left(A_{\mathrm{m}}\right)$, the product running over all maximal ideals of $R$, and $I(A)$ being the group of invertible fractional ideals of $A$ in $A \otimes K$.

(ii) Picent $\left(A_{\mathrm{m}}\right)=1$ for almost all $\mathrm{m}$ (i.e. with at most a finite number of exceptions).

(iii) The sequence

$$
1 \rightarrow \text { Picent }(\text { cent }(A)) \rightarrow \text { Picent }(A) \rightarrow \coprod_{\mathfrak{m}} \text { Picent }\left(A_{\mathfrak{m}}\right) \rightarrow 1
$$

is exact.

Remark. By Corollary 3 to Theorem 4 , the $A_{m}$ can be taken as local completions rather than localisations.

Proof. (i) If $Y \in I(A)$ then $Y_{\mathrm{m}}=A_{\mathrm{m}}$ for almost all $\mathrm{m}$. Thus the localisation map $I(A) \rightarrow \Pi I\left(A_{\mathrm{m}}\right)$ actually goes into $\amalg I\left(A_{\mathrm{m}}\right)$. It is injective, as $Y_{\mathrm{m}}=X_{\mathrm{m}}$, for all $\mathrm{m}$, implies $Y=X$, whenever $Y$ and $X$ are $R$-lattices in $A \otimes K$. On the other hand, there is an $R$-lattice $Y$ in $A \otimes K$ with given localisations $Y_{\mathrm{m}}$, provided that $Y_{\mathfrak{m}}=A_{\mathfrak{m}}$ for almost all $\mathrm{m}$. If $Y_{\mathfrak{m}}$ lies in $I\left(A_{\mathfrak{m}}\right)$ for all $\mathrm{m}$, then $Y$ lies in $I(A)$. Thus we have (i).

(ii) Let $A^{e}=A \otimes_{\text {cent }(A)} A^{\text {op }}$ be the enveloping algebra of $A$ and consider the homomorphism $l: \operatorname{Hom}_{A} e^{\left(A, A^{e}\right)} \rightarrow \operatorname{Hom}_{A} e^{(A, A)=\operatorname{cent}(A) \text {. This commutes with }}$ flat ring extension, and the surjectivity of $l$ is necessary and sufficient for $A$ to be separable over cent $(A)$. Thus $l \otimes_{\text {cent }(A)}\left(\operatorname{cent}(A) \otimes_{R} K\right)=l \otimes_{R} K$ is surjective, hence $l_{\mathrm{m}}$ is surjective for almost all $\mathrm{m}$, i.e. $A_{\mathrm{m}}$ is separable over cent $\left(A_{\mathrm{m}}\right)$, and so $\operatorname{Picent}\left(A_{\mathrm{m}}\right) \cong \operatorname{Picent}\left(\operatorname{cent}\left(A_{\mathrm{m}}\right)\right)=1$ for almost all $\mathrm{m}$, cent $\left(A_{\mathrm{m}}\right)$ being semilocal. [This argument simplifies if one assumes $A \otimes K$ to be separable over $K$.]

(iii) By part (i) (ii), and Corollary 2 to Theorem 4, we get a commutative diagram 


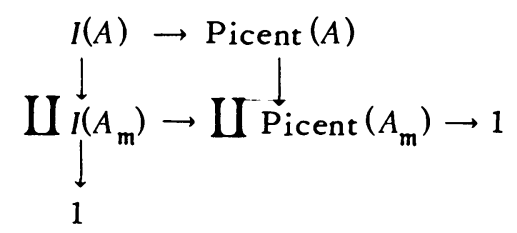

with exact rows and columns. Hence the right-hand column is surjective. The remainder of (iii) follows from Theorem 5 .

For a closer study of localisation it is natural to introduce idele groups. We define $\bar{U}(A)=\Pi_{\mathfrak{m}} U\left(\bar{A}_{\mathrm{m}}\right)$, where $\bar{A}_{\mathfrak{m}}$ is the completion of $A_{\mathfrak{m}}$. Next $J(A)=$ $\Pi_{\mathfrak{m}}\left(U\left(\bar{A}_{\mathfrak{m}} \otimes K\right) ; U\left(\bar{A}_{\mathfrak{m}}\right)\right)$ is the product of the groups $U\left(\bar{A}_{\mathfrak{m}} \otimes K\right)$, restricted with respect to the subgroups $U\left(\bar{A}_{\mathrm{m}}\right)$, i.e. the subgroup of $\Pi_{\mathrm{m}} U\left(\bar{A}_{\mathrm{m}} \otimes K\right)$ of elements $u$ with $u_{\mathfrak{m}} \in U\left(\bar{A}_{\mathfrak{m}}\right)$ for almost all $m$ (i.e. with at most a finite number of exceptions). $J(A)$ is given the topology for which $\bar{U}(A)$ is an open subgroup, and the subgroup topology on $\bar{U}(A)$ coincides with the product topology of $\Pi_{\mathrm{m}} U\left(\bar{A}_{\mathrm{m}}\right)$. These definitions fit into the context of adele groups of algebraic groups. If $K$ is a global field, then for deeper properties one has to take into account the "infinite primes" (i.e. those not coming from ideals of $R$ ). For the moment those can be neglected. Alternatively one can, for every infinite prime $m$, define $U\left(\bar{A}_{\mathfrak{m}}\right)=U\left((\overline{A \otimes K})_{\mathfrak{m}}\right)$, where $(\overline{A \otimes K})_{\mathrm{m}}$ is the completion at $\mathrm{m}$.

$U(A \otimes K)$ is embedded diagonally in $J(A)$. We shall also consider $J$ (cent $(A)$ ) as embedded in $J(A)$ (componentwise). Finally the idele normaliser of $A$ is $\bar{N}(A)=$ $J(A) \cap \Pi_{\mathfrak{m}} N\left(\overline{A_{\mathfrak{m}}}\right)$. Thus $\bar{N}(A)$ is (algebraically and topologically) the product of the groups $N\left(\bar{A}_{\mathrm{m}}\right)$ restricted with respect to the subgroups $U\left(\bar{A}_{\mathrm{m}}\right)$. As $U\left(\bar{A}_{\mathrm{m}}\right)$ is open in $N\left(\bar{A}_{\mathrm{m}}\right)$ and $N\left(A_{\mathrm{m}}\right)$ dense in $N\left(\bar{A}_{\mathrm{m}}\right)$, we have

$$
N\left(A_{\mathfrak{m}}\right) / U\left(A_{\mathfrak{m}}\right) \cong N\left(\bar{A}_{\mathfrak{m}}\right) / U\left(\bar{A}_{\mathfrak{m}}\right) .
$$

As $U\left(\operatorname{cent}\left(A_{\mathrm{m}} \otimes K\right)\right)$ is dense in $U\left(\operatorname{cent}\left(\bar{A}_{\mathrm{m}} \otimes K\right)\right)$, it follows by Corollary 2 to Theorem 4 that

$$
\bar{N}(A) / \bar{U}(A) J(\operatorname{cent}(A)) \cong \coprod_{\mathbf{m}} \operatorname{Outcent}\left(A_{\mathbf{m}}\right)
$$

Now we have

Corollary 1. There is a commutative diagram with exact rows and injective columns

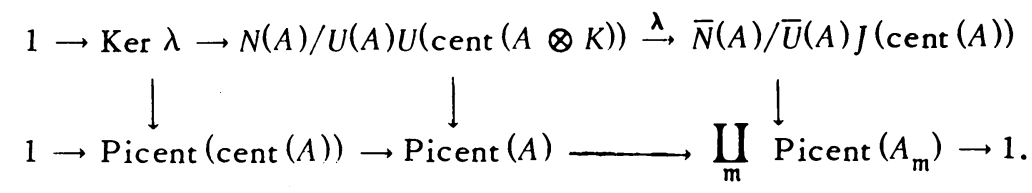

We shall derive a useful variant of this diagram. A left $A$-module $X$ is locally 
free if $X_{\mathrm{m}}$ is free of constant rank over $A_{\mathrm{m}}$, for all m. For an invertible $A-A$-bimodule $X$ over cent $(A)$ this amounts to saying that the image of $(X)$ under the localisation map falls into the subgroup $\amalg_{\mathfrak{m}} \operatorname{Outcent}\left(A_{\mathfrak{m}}\right)$ of $\amalg_{\mathfrak{m}}\left(\operatorname{Picent}\left(A_{\mathrm{m}}\right)\right)$. These classes $(X)$ form a subgroup of $\operatorname{Picent}(A)$ to be denoted by $\operatorname{LFPicent}(A)$, which coincides with $\mathrm{Picent}(A)$ if $A$ is a clean order (cf. [ST]). From Corollary 1 we now have

Corollary 2. There is a commutative diagram with exact rows and columns

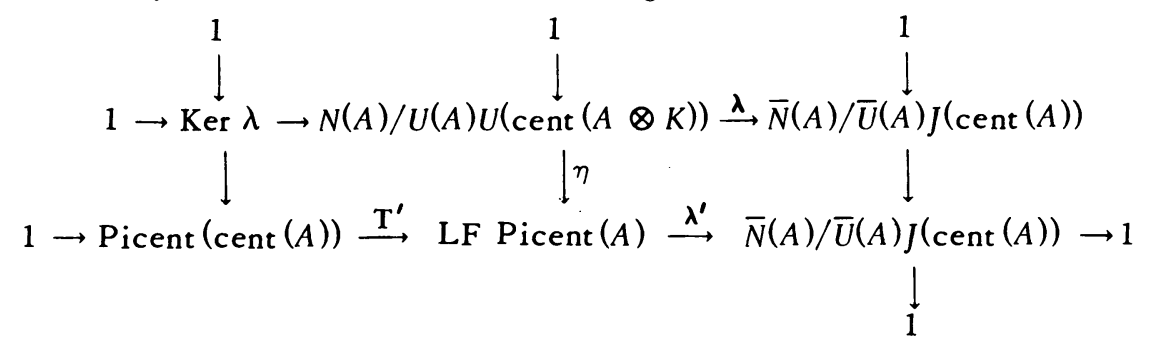

$\operatorname{Ker} \lambda$ measures the deviation from the Hasse principle for Outcent $(A)$. On the other hand the coset space

$$
\text { Coker } \lambda=[\bar{N}(A) / \bar{U}(A) J(\text { cent }(A))] / \operatorname{Im} \lambda
$$

measures the obstruction to local approximation. A similar interpretation also applies to the coset space

$$
\text { Coker } \eta=\operatorname{LFPicent}(A) / \text { Outcent }(A)=\operatorname{LFPicent}(A) / \operatorname{Im} \eta
$$

which in the first place, as a subset of $\operatorname{Proj}(A)$ (cf. Theorem 1) was defined as a set of left $A$-module isomorphism classes. In fact the maps $\bar{N}\left(\bar{A}_{\mathrm{m}}\right) \rightarrow I\left(\bar{A}_{\mathrm{m}}\right)$ (cf. Theorem 4) yield an injective homomorphism $\bar{N}(A) / \bar{U}(A) \rightarrow \amalg I\left(\bar{A}_{\mathrm{m}}\right) \cong I(A)$, in whose image are exactly those fractional ideals, whose class falls into LFPicent $(A)$. Identifying $\bar{N}(A) / \bar{U}(A)$ with its image in $I(A)$ we get a commutative diagram

$$
\begin{aligned}
1 & \rightarrow U(\operatorname{cent}(A \otimes K)) / U(\operatorname{cent}(A)) \rightarrow N(A) / U(A) \\
\| & \rightarrow N(A) / U(A) J(\text { cent }(A)) \rightarrow 1 \\
1 & \rightarrow U(\operatorname{cent}(A \otimes K)) / U(\operatorname{cent}(A)) \rightarrow \bar{N}(A) / \bar{U}(A) \rightarrow \text { LFPicent }(A) \rightarrow 1 .
\end{aligned}
$$

Thus indeed

$$
\text { Coker } \eta=\operatorname{Coker}[N(A) / U(A) \rightarrow \bar{N}(A) / \bar{U}(A)]
$$

(coset spaces). As $\eta$ is injective so is $N(A) / U(A) \rightarrow \bar{N}(A) / \bar{U}(A)$ i.e. the Hasse principle holds for $N(A) / U(A)$.

Via the map $\mathrm{T}^{\prime}$, Picent (cent $\left.(A)\right)$ is identified with a central subgroup of LFPicent $(A)$. Its action by, say, left translation yields an action of Picent(cent $(A)$ ) as permutation group on LFPicent $(A) /$ Outcent $(A)=$ Coker $\eta$. We shall now obtain a characterisation of $\operatorname{Ker} \lambda$ and of $\operatorname{Coker} \lambda$ in terms of this action. Clearly the composite map 


$$
\text { LFPicent }(A) \rightarrow \bar{N}(A) / \bar{U}(A) J(\text { cent }(A)) \rightarrow \text { Coker } \lambda
$$

factorises uniquely through a map

$$
\delta: \text { Coker } \eta \rightarrow \text { Coker } \lambda \text {. }
$$

We then have

Theorem 7. (i) $\operatorname{Ker} \lambda$ is the stabiliser of every element of Coker $\eta$ under the action of Picent(cent $(A))$.

(ii) $\delta$ induces a bijection of the set of orbits of Coker $\eta$ under the action of Picent (cent $(A))$ onto Coker $\lambda$.

Proof. A straightforward bit of diagram chasing. In fact Theorem 7 arises from an application of a general non-Abelian snake lemma!

6. Radical reduction. The localisation theorems of $\$ 4$ and $\$ 5$ almost reduce the study of Picent to the commutative and the local case. In the next two sections we shall develop results and techniques relevant for the local case, although the contents of the present section is somewhat more general.

A radical ideal $\Re$ of $A$ is an ideal of $A$ contained in the Jacobson radical $\left({ }^{2}\right)$ $J(A)=J$ of $A$ and so that, for all invertible $A$-A-bimodules $Y, \Re Y=Y \Re$. Then. $A / \Re \otimes_{A} Y=Y \otimes_{A} A / \Re$ is an invertible $A / \Re-A / \Re$-bimodule and we get a homomorphism

$$
\rho_{\mathfrak{R}}: \operatorname{Pic}_{R}(A) \rightarrow \operatorname{Pic}_{R}(A / \mathcal{R}),
$$

which can be useful for computing $\operatorname{Pic}_{R}(A)$ and which will be studied here. The main application is to $\Re=J(A)^{r}$ when $A$ is a finite dimensional algebra over a field or a local order, and to $\Re=J(R)^{r} A$.

Recall the definition of an $R$-derivation $d: B \rightarrow L$, where $B$ is an $R$-algebra and $L$ a $B$-B-bimodule. $d$ is an $R$-linear map so that $d\left(b_{1} b_{2}\right)=b_{1} d\left(b_{2}\right)+d\left(b_{1}\right) b_{2}$. With each $l \in L$ one associates the inner derivation $d_{l}$ given by $d_{l}(b)=b l-l b$. The map $l \mapsto d_{l}, L \rightarrow \operatorname{Der}_{R}(B, L)(R$-module of $R$-derivations $B \rightarrow L)$ is $R$-linear, and its cokernel is the $R$-module $H^{1}(B, L)$.

We shall denote by $A_{u t}(A ; \Re)$ the subgroup of $A_{u_{t}}(A)$ of automorphisms $f$ with $f \Re=\Re$, and which induce the identity automorphism on $A / \Re$.

Theorem 8. Let $\Re$ be a radical ideal of $A$. Then we bave an exact sequence

$$
\operatorname{Aut}_{R}(A ; \Re) \rightarrow \mathrm{Pic}_{R}(A) \stackrel{\rho_{\mathfrak{R}}}{\rightarrow} \mathrm{Pic}_{R}(A / \Re)
$$

where the left-hand map comes from $\Omega$ (cf. Theorem 1). If moreover $\Re \subset J(A)^{2}$, $J(A) \Re+\Re J(A)=0$, then we bave an exact sequence

$\left({ }^{2}\right)$ Note that we are from now on using the symbol $J$ in an entirely different meaning from that in $\$ 5$. No confusion should arise. 


$$
H^{1}\left(A / J(A)^{2}, \Re\right) \rightarrow \mathrm{Pic}_{R}(A) \rightarrow \mathrm{Pic}_{R}(A / \Re) .
$$

Proof. If $f \in \operatorname{Aut}_{R}(A ; \Re)$ then clearly $A / \Re \otimes_{A} A f \cong A / \Re$ as bimodules. Conversely let $(X) \in \operatorname{Ker} \rho_{\Re}$. We are thus given an isomorphism $f: A / \Re \cong X \otimes_{A} A / \Re$, of bimodules. We can lift $f$ to an isomorphism $\bar{f}: A \cong X$ of left $A$-modules. By Theorem 1 there is an automorphism $g$ of $A$ with $\bar{f}(a)=\bar{f}(1) g(a), \forall a \in A$. By hypothesis $g(a)-a \in \Re$, i.e. $g \in \operatorname{Aut}_{R}(A ; \Re)$, as we had to show.

With the additional hypothesis on $\Re$ one gets an $R$-linear map $\operatorname{Der}_{R}\left(A / J^{2}, \Re\right) \rightarrow$ $\operatorname{Aut}_{R}(A ; \Re)$ by first lifting a derivation $A / J^{2} \rightarrow \Re$ to one $d: A \rightarrow \Re$ and then defining $f_{d}(a)=a+d(a)$. Then $f_{d} \in \mathrm{Aut}_{R}(A ; \Re)$. One easily sees that this yields an isomorphism. The inner derivations map precisely onto the inner automorphisms induced by elements in $1+\Re$. By Theorem 1 , we now get the required result.

Corollary 1. If $A$ is a finite-dimensional algebra over a field, or an order over a discrete valuation ring, and if $\operatorname{cent}(A) \rightarrow \operatorname{cent}(A / J)(J=J(A))$ is surjective then Autcent $(A) \rightarrow \operatorname{Picent}(A)$ is surjective.

For, we can apply the theorem with $R=\operatorname{cent}(A)$, and just use the fact that $\operatorname{Picent}(A / J)=1$.

Corollary 2. If $A$ is a finite ring then $\mathrm{Pic}(A)$ is finite. If moreover the additive group of $A$ is a p-group then $\operatorname{Ker}\left[\mathrm{Pic}(A) \rightarrow \mathrm{Pic}\left(A / J^{2}\right)\right]$ is a p-group.

Proof. Take $R=\mathrm{Z}$. As $A / J$ is semisimple Picent $(A / J)=1$, hence, by Theorem 2, $\operatorname{Pic}(A / J)$ is embedded in the finite group Aut $_{\mathrm{Z}}$ (cent $(A / J)$ ). Also Aut $_{\mathrm{Z}}(A)$ is finite, and so by Theorem $8 \mathrm{Pic}_{\mathrm{Z}}(A)$ is finite.

Let $L_{n}=\operatorname{Ker}\left[\operatorname{Pic}\left(A / J^{n}\right) \rightarrow \operatorname{Pic}\left(A / J^{2}\right)\right]$. Under the hypothesis that $A$ is a $p$ group we show, by induction on $n \geq 2$, that $L_{n}$ is a $p$-group. As $J^{n}=0$, for some $n$, the required result will follow.

The assertion is trivial for $n=2$. For $n>2$ we get a homomorphism $L_{n} \rightarrow$ $L_{n-1}$ whose kernel is embedded in $\operatorname{Ker}\left[\operatorname{Pic}\left(A / J^{n}\right) \rightarrow \operatorname{Pic}\left(A / J^{n-1}\right)\right]$ and it only remains to show that the latter is a $p$-group. Here we take $R=(\mathrm{Z} / p) \mathrm{Z}^{m}, m$ large enough. Then we can apply the second part of Theorem 8 , with $A$ replaced by $A / J^{n}$ and with $\Re=J^{n-1} / J^{n}$. We conclude that $\operatorname{Ker}\left[\operatorname{Pic}\left(A / J^{n}\right) \rightarrow \operatorname{Pic}\left(A / J^{n-1}\right)\right]$ is a quotient of $H^{1}\left(A / J^{2}, J^{n-1} / J^{n}\right)$ which is an $R$-module, hence a $p$-group.

Corollary 3. Let $R$ be a discrete valuation ring with quotient field $K, A$ an order over $R$ in a semisimple algebra $A \otimes K$. Suppose that cent $(A) \rightarrow \operatorname{cent}(A / J)$ is surjective. If $N_{1}(A)$ is the subgroup of the normaliser $N(A)$ of $A$ in $A \otimes K$, of elements $u$ with $u a u^{-1}=a(\bmod J), \forall a \in A$, then $N(A)=N_{1}(A) U(A)$.

Proof. By the theorem, Autcent $(A ; J) \rightarrow \operatorname{Picent}(A)$ is surjective, i.e. has the same image as Autcent $(A) \rightarrow \operatorname{Picent}(A)$. Translate this into a statement on $N(A)$, to get the corollary. 


\section{Local orders.}

Notation. $R$ is a discrete valuation ring with quotient field $K$ and maximal ideal $h, R / f=k, R / p^{r}=k_{r} \cdot A$ is an order over $R$ and $A \otimes K$ is separable over $K$. The unadorned $\otimes$ is over $R$.

$$
\text { Let, for } s \geq 1 \text {, }
$$

$$
T_{s}=\left[a \in A \mid a x-x a \in p^{s} A, \forall x \in A\right]
$$

We then have

Theorem 9. $\exists r$, so that $T_{r} \subset \operatorname{cent}(A)+\wp A$, and, for any sucb $r, \operatorname{Picent}(A) \rightarrow$ $\mathrm{Pic}_{R}\left(A \otimes k_{r}\right)$ is injective.

We first prove

Lemma C. $\exists t$, so that, for all $s, T_{t+s} \subset \operatorname{cent}(A)+\hbar^{s} A$.

Proof of the lemma. The inclusion will be shown to hold whenever $b^{t} H^{1}(A, A)=0$. One knows there is such a $t$, the least value giving the Higman ideal (cf. [DH]).

In fact if $a \in T_{t+s}$ then there is a derivation $d$ of $A$ so that $a x-x a=$ $\pi^{s+t} d(x), \forall x \in A$, where $\pi \in R, \pi R=\uparrow$. But then by hypothesis $\pi^{t} d$ is principal, i.e., for all $x, \pi^{t} d(x)=b x-x b$, for some fixed $b \in A$. Hence $a-\pi^{s} b \epsilon$ $\operatorname{cent}(A)$, and this is what we had to show.

Proof of the theorem. The first part follows from the lemma, taking $s=1$.

For the second part recall that $\operatorname{Picent}(A)=\operatorname{Ker}\left[\mathrm{Pic}_{R}(A) \rightarrow \operatorname{Pic}(A \otimes K)\right]$ (cf. Corollary 2 to Theorem 4). We have to show then that the kernel of

$$
\mathrm{Pic}_{R}(A) \rightarrow \mathrm{Pic}(A \otimes K) \times \mathrm{Pic}\left(A \otimes k_{r}\right)
$$

is null, if $r$ satisfies the stated condition.

Consider a bimodule $Y$ whose class falls into this kernel. Then $A \otimes K \cong$ $Y \otimes K$ as bimodules, whence $C \otimes K \cong Y^{A} \otimes K$, where we shall write $C=\operatorname{cent}(A)$. Thus

$$
C \text { and } Y^{A} \text { are free } R \text {-modules of the same rank. }
$$

Next let

$$
T_{r}(Y)=\left[y \in Y \mid y x-x y \in \mathbb{R}^{r} Y, \forall x \in A\right] .
$$

We are given an isomorphism $g: A \otimes k_{r} \cong Y \otimes k_{r}$ of bimodules giving rise to is omorphisms

$$
p\left(A \otimes k_{r}\right) \cong p\left(Y \otimes k_{r}\right)
$$

and 


$$
T_{r} / \mathfrak{p}^{r} A=\left(A \otimes k_{r}\right)^{A} \cong\left(Y \otimes k_{r}\right)^{A}=T_{r}(Y) / \mathfrak{p}^{r} Y
$$

and hence to a commutative diagram

$$
\begin{aligned}
& 0 \rightarrow \mathfrak{p} A / \mathfrak{p}^{r} A \rightarrow T_{r}+\mathfrak{p} A / \mathfrak{p}^{r} A \longrightarrow T_{r}+\mathfrak{p} A / \mathfrak{p} A \rightarrow 0 \\
& 0 \rightarrow \mathfrak{p} Y / \mathfrak{p}^{r} Y \rightarrow T_{r}(Y)+\mathfrak{p} Y / \mathfrak{p}^{r} Y \rightarrow T_{r}(Y)+\mathfrak{p} Y / \mathfrak{p} Y \rightarrow 0
\end{aligned}
$$

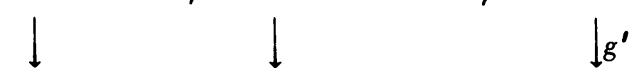

with exact rows and bijective columns. By hypothesis on $r, T_{r}+p A / p A=$ $C+\not A / \hbar A \cong C / \hbar C$, and hence $\operatorname{dim}_{k}\left(T_{r}(Y)+\wp Y / \hbar Y\right)=\operatorname{dim}_{k}(C / \not C)$, and the latter coincides by (7.2) with $\operatorname{dim}_{k}\left(Y^{A} / p Y^{A}\right)=\operatorname{dim}_{k}\left(Y^{A}+\wp Y / p Y\right)$. (We are using the fact that $Y^{A}$ is pure in $Y$.) But $Y^{A}+\wp Y / p Y \subset T_{r}(Y)+\wp Y / p Y$ and hence in fact, on comparing dimensions,

$$
Y^{A}+p Y / p Y=T_{r}(Y)+p Y / p Y .
$$

Thus the isomorphism $g^{\prime}$ (last column of (7.4)) is really an isomorphism $C / P C \cong$ $Y^{A} / p Y^{A}$, of $C$-modules, lifting to a homomorphism $C \rightarrow Y^{A}$ which by Nakayama's Lemma is surjective, and so by (7.2) is bijective, i.e. we have

$$
C \cong Y^{A}
$$

as $C$-modules.

Now observe that in view of the given isomorphism $A \otimes k_{r} \cong Y \otimes k_{r}$, the map $w_{Y \otimes k_{r}}:\left(Y \otimes k_{r}\right)^{A} \otimes_{C \otimes k_{r}} A \otimes k_{r} \rightarrow Y \otimes k_{r}$ is an isomorphism, i.e. the pairing

$$
T_{r}(Y) / \mathfrak{p}^{r} Y \otimes A / \mathfrak{p}^{r} A \rightarrow Y / \mathfrak{p}^{r} Y
$$

is surjective, hence a fortiori

$$
T_{r}(Y)+\not p Y / \mathfrak{p} Y \otimes A / \mathfrak{p} A \rightarrow Y / \mathfrak{p} Y
$$

is. But then, by (7.5), the pairing

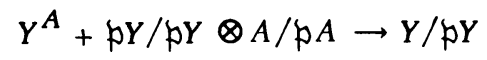

is surjective. As $Y^{A}+\wp Y / p Y \cong Y^{A} / \hbar Y^{A}$ it follows, by Nakayama's Lemma, that (with $w_{Y}$ as in $\$ 4$, Lemma B) $w_{Y}: Y^{A} \otimes_{C} A \rightarrow Y$ is surjective. As $w_{Y} \otimes K=$ $w_{Y \otimes K}$ is bijective, $w_{Y}$ is injective, hence bijective and so by $(7 . \dot{6})$

$$
A \cong C \otimes_{C} A \cong Y^{A} \otimes_{C} A \cong Y
$$

as bimodules, as we had to show.

Corollary 1. If $k$ is finite then $\operatorname{Picent}(A)$ is finite. 
Proof. By the theorem and by Corollary 2 to Theorem 8 .

Corollary 2. If $k$ is finite then $N(A) / U(A) U(\operatorname{cent}(A \otimes K)$ ) is finite.

For, by Corollary 2 to Theorem 4 this group embeds in Picent $(A)$.

Corollary 3. If $k$ is finite of characteristic $p$ then $\operatorname{Ker}\left[\operatorname{Picent}(A) \rightarrow \operatorname{Pic}_{R}\left(A / J^{2}\right)\right]$ is a p-group.

Proof. We may choose $r>1$ in Theorem 9. Then $A / J^{2}=\left(A / J^{2}\right) \otimes k_{r}=$ $\left(A \otimes k_{r}\right) /\left(J\left(A \otimes k_{r}\right)\right)^{2}$. We now get, by Theorem 9, a commutative diagram with exact row

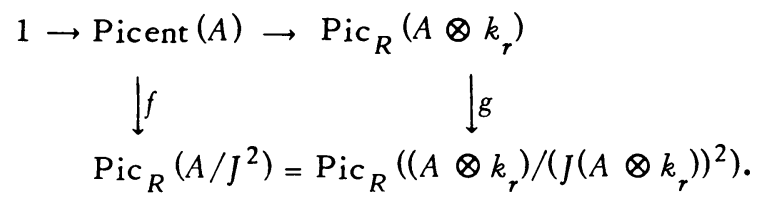

By Corollary 2 to Theorem $8, \operatorname{Ker} g$ is a p-group. Hence so is its subgroup $\operatorname{Ker} f$.

Corollary 4. For some $t>0, \operatorname{Pic}_{R}(A) \rightarrow \operatorname{Pic}\left(A \otimes k_{t}\right)$ is injective.

Proof. Choose $t$ so that, as in the theorem. $\operatorname{Picent}(A) \rightarrow \operatorname{Pic}\left(A \otimes k_{t}\right)$ is injective, and that moreover $\operatorname{Aut}_{R}(\operatorname{cent}(A)) \rightarrow A_{u t}\left(\operatorname{cent}(A) \otimes k_{t}\right)$ is injective, which can clearly be achieved; as $\operatorname{Aut}_{R}(\operatorname{cent}(A))$ is a finite group. If now $Y$ is an invertible $A$-A-bimodule over $R$ so that $Y \otimes k_{t} \cong A \otimes k_{t}$ as bimodules, then $Y \otimes k_{t}$ is "over" cent $\left(A \otimes k_{t}\right)$. As cent $(A) \otimes k_{t}$ embeds in cent $\left(A \otimes k_{t}\right)$ the automorphism $\Phi(Y) \otimes k_{t}$ is trivial, hence $\Phi(Y)$ is trivial, hence $(Y) \in \operatorname{Picent}(A)$, and thus by the Theorem $Y \cong A$ as bimodule.

Remark. There is a quick direct proof of the last corollary, along lines suggested by I. Reiner and S. Ullom. One takes $t>s$, where $h^{s}$ is the Higman ideal. An isomorphism $A \otimes k_{t} \cong Y \otimes k_{t}$ of bimodules lifts to an isomorphism $f: A \cong Y$ of left $A$-modules. Viewing $\operatorname{Hom}\left({ }_{A} Y,{ }_{A} A\right)$ as an $A-A$-bimodule via the right $A$ module structures of $Y$ and $A$, we moreover see that there is a derivation $d$ of $A$ in $\operatorname{Hom}\left({ }_{A} Y, A_{A}^{A}\right)$ so that, with $\pi \in R, \pi R=p$, we have $f a-a f=\pi^{t} d(a)$. Moreover $\pi^{s} d$ is principal, say induced by $g$, and then $f-\pi^{t-s} g$ is a bimodule homomorphism $A \rightarrow Y$, and as $t-s>0$ is still an isomorphism.

I have retained Theorem 9 in its present form however as the actual minimal value of $r$ does matter. Thus if $A=R(\Omega)$ is a group ring of a finite group $\Omega$ then we may take $r=1$ and so $\operatorname{Picent}(A) \rightarrow \operatorname{Pic}(A \otimes k)$ is injective, while the Higman ideal is $R(\operatorname{card} \Gamma)$.

Topological interpretation of Lemma C. $U(A \otimes K)$ as a linear group over the valued field $K$ has a topology, and $U(C \otimes K)$ is a closed subgroup. We can then define on Autcent $(A \otimes K)$ the quotient topology, via the identification $U(A \otimes K) / U(C \otimes K) \cong$ Autcent $(A \otimes K)$. On the other hand Autcent $(A \otimes K)$ is a closed 
subgroup of the linear group of linear automorphisms of the $K$-vector space $A \otimes K$, and as such has a subgroup topology. Then we have immediately

Coroll ary to Lemma C. The two topologies coincide.

8. Commutative orders. The localisation theorem for orders largely reduces the computation of Picent $(A)$ to two special cases, namely the local one and the commutative one. Thus the results of [F] for commutative orders are in fact of wider interest, and for convenience and completeness sake we restate some of these here. Throughout $R$ is a Dedekind domain with quotient field $K . A$ is a commutative order over $R$ with $A \otimes K=\Pi_{i} L^{(i)}$ where the $L^{(i)}$ are separable extension fields of $K$. The maximal order $M$ of $A \otimes K$ over $R$ is then the product $M=\Pi_{i} M^{(i)}$ of the integral closures $M^{(i)}$ of $R$ in $L^{(i)}$.

Theorem 10. There is an exact sequence

$$
1 \rightarrow U(M) / U(A) \rightarrow \prod_{\mathrm{m}}^{*} U\left(M_{\mathrm{m}}\right) / U\left(A_{\mathrm{m}}\right) \rightarrow \operatorname{Picent}(A) \rightarrow \prod_{i} C\left(M^{(i)}\right) \rightarrow 1,
$$

where $C\left(M^{(i)}\right)=\operatorname{Picent}\left(M^{(i)}\right)$ is the ideal class group of $M_{i}$, and where $\Pi^{*}$ extends over a set of maximal ideals $m$ containing all thoses with $M_{m} \neq A_{m}$. Hence if we define

$$
D(A)=\operatorname{Ker}\left[\operatorname{Picent}(A) \rightarrow \prod_{i} C\left(M^{(i)}\right)\right]
$$

then

$$
D(A) \cong \prod_{m}^{*} U\left(M_{m}\right) / \prod_{m}^{*} U\left(A_{m}\right) \cdot U(M)
$$

If the residue class fields of $R$ are all finite then we get for the group order

$$
\operatorname{card}(D(A))=\prod_{\mathfrak{m}}^{*}\left[U\left(M_{\mathfrak{m}}\right): U\left(A_{\mathfrak{m}}\right)\right] /[U(M): U(A)]
$$

where $[X: Y]$ is the group index. If $p$ is the characteristic of $R / m$ then the $p$ part of $\left[U\left(M_{\mathfrak{m}}\right): U\left(A_{\mathfrak{m}}\right)\right]$ is $\left[J\left(M_{\mathfrak{m}}\right): J\left(A_{\mathfrak{m}}\right)\right]$ (Jacobson radicals) and the p-prime part is

$$
\operatorname{card}\left(U\left(M_{\mathfrak{m}} / J\left(M_{\mathfrak{m}}\right)\right)\right) / \operatorname{card}\left(U\left(A_{\mathfrak{m}} / J\left(A_{\mathfrak{m}}\right)\right)\right)
$$

Proof (cf. [F]).

9. Finiteness. We take $R=Z$. It is obvious that analogous results hold whenever class numbers for integral closures in algebraic field extensions and residue class fields are finite. 
Theorem 11. If $A$ is a Z-order with $A \otimes Q$ semisimple, then $\operatorname{Pic}(A)$ is finite.

Proof. By Theorem 2, $\operatorname{Pic}(A) / \operatorname{Picent}(A)$ embeds in the finite group

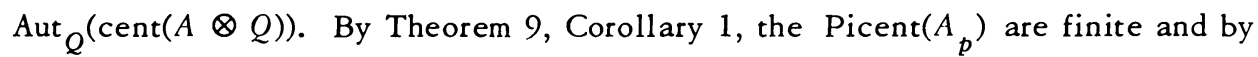
Theorem 10 Picent $(c e n t(A))$ is finite. Hence by Theorem 6 Picent $(A)$ and thus $\operatorname{Pic}(A)$ are finite. The theorem is of course a consequence of the Jordan-Zassenhaus Theorem, but as seen we have a short direct proof.

10. Maximal orders. Here $R$ is a Dedekind domain with quotient field $K, B$ is a finite dimensional separable $K$-algebra. In the example of $\$ 2$ we showed how to compute $\mathrm{Pic}_{K}(B)=$ Aut $_{K}\left(\operatorname{cent}(B) \mid M_{-} \operatorname{2Mg}(B)\right)$ by looking at the effect of automorphisms on Brauer groups.

Theorem 12. Let $A$ be a maximal order in $B$. Then

(i) Any maximal order $A^{\prime}$ in $B$ is Morita equivalent to $A$, and all such Morita equivalences yield the same isomorphism $\operatorname{Picent}\left(A^{\prime}\right) \cong \operatorname{Picent}(A)$.

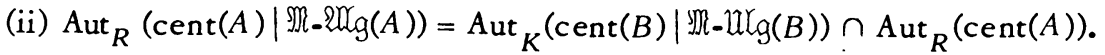

(iii) All invertible A-A-bimodules are locally free (always as left $A$-modules).

(iv) $I(A)$ is a free Abelian group on the maximal two-sided ideals of $A$. Picent $(A)$ is an Abelian group.

Now suppose moreover that $B$ is central simple over $K$. Then

(v) For every maximal ideal $\mathrm{m}$ of $R, I\left(A_{\mathrm{m}}\right)$ is free Abelian on the radical $J\left(A_{\mathfrak{m}}\right)$, and, for $\mathrm{m} A_{\mathfrak{m}}=J\left(A_{\mathfrak{m}}\right)^{e_{\mathfrak{m}}}$, we have Picent $\left(A_{\mathfrak{m}}\right)=\mathrm{Z} / e_{\mathfrak{m}} \mathbf{Z}$.

(vi) With $\lambda=\lambda_{A}$ as in Corollary 1 to Theorem $6,\left(\operatorname{Ker} \lambda_{A}\right)^{n}=1$ where $\operatorname{dim}_{K}(B)=$ $n^{2}$, and $\left(\operatorname{Cok} \lambda_{A}\right)^{e}=1$, e the lowest common multiple of the $e_{\mathrm{m}}$. Hence $\left(\operatorname{Cok} \lambda_{A}\right)^{n}=1$.

[Note that $\operatorname{Cok} \lambda_{A}$ is a group, as $\amalg_{m} \operatorname{Picent}\left(A_{m}\right)$ is Abelian.]

One can obtain rather stronger results than (vi), in the context of a change of orders from $A$ to $M_{r}(A)$ ( $r$ by $r$ matrices over $A$ ), i.e. involving the application of Theorem 1, (ii) and (iii). It is however tidier to discuss this when the additional result of the subsequent note [FRU] is at our disposal.

A great deal of the Theorem is known in some form or other, specifically the Morita equivalence $A \sim A^{\prime}$ in (i), also (ii), (iii), (iv) and (v). (See e.g. [R], [SW], [RHD].) We shall therefore only give a brief outline, indicating how this special case fits into our general theory. We shall assume certain basic results of Morita theory, plus (a) the valuation theory for a division algebra over the quotient field of a complete discrete valuation ring $R$ (cf. [DE]), (b) the fact that locally any two maximal orders of a separable algebra are conjugate (cf. [R]), (c) that in the local case with $B$ simple any finitely generated $A$-module, torsion free over $R$ is a direct sum of copies of a unique indecomposable such $A$-module (cf. [R]). 
(c) immediately implies (iii). (b) implies that $A^{\prime}$ and $A$ are Morita equivalent, and the remainder of (i) then follows from the fact that Picent $(A)$ is Abelian. Moreover by (i) and Morita theory, any two maximal orders $A$ and $A$ ' of Morita equivalent simple algebras $B$ and $B^{\prime}$ are Morita equivalent. This is easily seen to imply (ii).

(iv) will follow from (v). To prove (v) we can suppose that $R$ is complete local and, by Morita theory, that $B$ is a division algebra. Then the result follows from valuation theory.

Finally for (vi) first observe that $\operatorname{Cok} \lambda_{A}$, as a quotient of $\amalg \operatorname{Picent}\left(A_{\mathrm{m}}\right)=$ II $\mathrm{Z} / e_{\mathrm{m}} \mathrm{Z}$, is annihilated by $e$. For the result on $\operatorname{Ker} \lambda_{A}$ one uses the reduced norm $\nu$. A straightforward local computation shows that the composition Picent(cent $(A)) \stackrel{\mathrm{T}}{\rightarrow} \operatorname{Picent}(A) \stackrel{\nu}{\rightarrow} \operatorname{Picent}(\operatorname{cent}(A))$ is just $(X) \mapsto(X)^{n}$. But Outcent $(A) \subset \operatorname{Ker} \nu$. For, the image under $\nu$ of the class of $A b(b \in N(A))$ is the class of the reduced norm of $b$, i.e. the principal class in Picent(cent $(A)$ ). Thus if $(X) \in \operatorname{Ker} \lambda_{A}$ then $(X)^{n}=\nu \mathrm{T}(X)=1$.

11. Congruence orders. These orders provide examples for a number of interesting phenomena-in particular a splitting of the localisation sequence, nonAbelian Picard groups and special properties of the normaliser.

$R$ and $K$ are as in the previous section. With each integer $n>0$ and each nonzero ideal $a$ of $R$ we associate a congruence order $A=R+a M_{n}(R) \subset M_{n}(K)$. Clearly $A$ spans $M_{n}(K)$. For a maximal ideal $m$ of $R, A_{\mathfrak{m}}=R_{\mathfrak{m}}+\mathrm{m}^{a} M_{n}\left(R_{\mathfrak{m}}\right)$, where $\mathrm{m}^{a}$ is the precise power of $m$ dividing $a$.

We shall write $\operatorname{PGL}(S, n)$ for the projective general linear group of a ring $S$ in dimension $n$, i.e. for the quotient of $\mathrm{GL}(S, n)$ module $U(S)$.

Theorem 13. (i) Every invertible A-A-bimodule is locally free.

(ii) $N(A)=N\left(M_{n}(R)\right)$. Hence $\mathrm{GL}(R, n) \subset N(A)$. The resulting map $\mathrm{GL}(R, n) \rightarrow$ Outcent $(A)$ is surjective if $R$ is a principal ideal domain.

(iii) $\operatorname{Picent}\left(A_{\mathfrak{m}}\right)=\operatorname{PGL}\left(R_{\mathfrak{m}} / \mathfrak{m}^{a}, n\right)\left(\mathfrak{m}^{a}\right.$ as above). Hence $\Pi_{\mathfrak{m}} \operatorname{Picent}\left(A_{\mathfrak{m}}\right)=$ $\operatorname{PGL}(R / a, n)$.

(iv) The localisation sequence $1 \rightarrow \operatorname{Picent}(R) \rightarrow \operatorname{Picent}(A) \rightarrow \operatorname{PGL}(R / a, n)$ $\rightarrow 1$ splits.

(v) The map $X \mapsto X M_{n}(R)$ defines a bomomorphism $I(A) \rightarrow I\left(M_{n}(R)\right)$ and the map $(X) \mapsto\left(X \otimes_{A} M_{n}(R)\right)((X) \in \operatorname{Picent}(A))$ a bomomorpbism into Picent $\left(M_{n}(R)\right)$ so that the diagram

commutes.

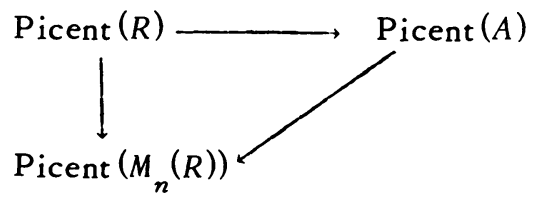


Proof. We shall first suppose that $R$ is a local ring with maximal ideal $\mathrm{m}$. The case $a=R$ is trivial, and so we assume $a=m^{a}$ with $a>0$. Then $A / J(A)=$ $R / \mathrm{m}$. As every $R / \mathrm{m}$-module is free, the same is true for every projective left $A$ module. Thus every invertible $A$-A-bimodule is free of rank 1 . This establishes (i).

For (ii), still keeping $R$ local, observe that clearly $N\left(M_{n}(R)\right) \subset N(A)$. Conversely one shows (e.g. by induction on $a$ ) that $T \in R+\mathrm{m}^{a} M_{n}(R)$, $\operatorname{det} T \in \mathrm{m}^{n a}$ is equivalent with $T \in \mathrm{m}^{a} M_{n}(R)$. Thus any normaliser of $A$ also normalises $\mathrm{m}^{a} M_{n}(R)$, hence $M_{n}(R)$. Thus $N(A)=N\left(M_{n}(R)\right)$. This result then holds also globally.

By (ii) we have $\mathrm{GL}(R, n) \subset N(A)$ for any $R$ and hence we get a map

$$
\rho: \mathrm{GL}(R, n) \rightarrow \text { Outcent }(A)=N(A) / U(A) U(K) .
$$

If $R$ is a principal ideal domain then $\operatorname{Outcent}\left(M_{n}(R)\right) \subset \operatorname{Picent}\left(M_{n}(R)\right)=\operatorname{Picent}(R)=1$, i.e. $N\left(M_{n}(R)\right)=\mathrm{GL}(R, n) U(K)$. Thus, by (ii), $\rho$ is surjective. In the local case Ker $\rho$ consists precisely of the matrices in $U(A)$, and hence now

$$
\text { Outcent }(A) \cong \mathrm{GL}(R, n) / U(A)=\operatorname{PGL}\left(R / \mathrm{m}^{a}, n\right) \text {. }
$$

By (i), this is also Picent $(A)$.

For (v) consider $X \in I(A)$. For every $m, X_{\mathfrak{m}}=A_{\mathfrak{m}} \alpha_{\mathfrak{m}}, \alpha_{\mathrm{m}} \in N\left(A_{\mathrm{m}}\right)$. As $\alpha_{\mathrm{m}} \in N\left(M_{n}\left(R_{\mathrm{m}}\right)\right)$, by (ii), we get

$$
X_{m} M_{n}\left(R_{m}\right)=M_{n}\left(R_{m}\right) X_{m}=M_{n}\left(R_{m}\right) \alpha_{m}=\alpha_{m} M_{n}\left(R_{m}\right),
$$

and thus $X M_{n}(R)=M_{n}(R) X$ is an $M_{n}(R)-M_{n}(R)$-bimodule, and thus an invertible fractional ideal of $M_{n}(R)$. It is clear that we get a homomorphism $I(A) \rightarrow I\left(M_{n}(R)\right)$. Going over to isomorphism classes we get, by Theorem 4 , the required homomorphism, clearly satisfying the stated commutativity condition.

The column in the diagram in (v) is an isomorphism. The resulting map Picent $(A) \rightarrow \operatorname{Picent}(R)$ thus splits the localisation sequence, as asserted under (iv).

Corollary. Cok $\lambda_{A}$ is a group and there is a surjective homomorphism of $\operatorname{Cok}\left[U(R) \rightarrow U(R / a) / U(R / a)^{n}\right]$ onto Cok $\lambda_{A}$, which is an isomorpbism if $R$ is a principal ideal domain.

Proof. We simply have to show that the image of $r: \mathrm{GL}(R, n) \rightarrow \operatorname{PGL}(R / a, n)$ is normal and that $\operatorname{Cok} \tau \cong \operatorname{Cok}\left[U(R) \rightarrow U(R / a) / U(R / a)^{n}\right]$.

One knows, as $R / a$ is a semilocal ring, that $\operatorname{SL}(R, n) \rightarrow \operatorname{SL}(R / a, n)$ is surjective, hence via the determinantal map $\operatorname{Cok}[\mathrm{GL}(R, n) \rightarrow \mathrm{GL}(R / a, n)] \cong$ $\operatorname{Cok}[\mathrm{U}(R) \rightarrow U(R / a)]$ with $\operatorname{Im}[\mathrm{GL}(R, n) \rightarrow \mathrm{GL}(R / a, n)]$ indeed a normal subgroup. The result is now immediate. 
12. Group rings. In this section we collect a number of results on group rings. We first consider the local groups $\operatorname{Picent}\left(A_{\mathrm{m}}\right)$, for group rings $A$. Let then $R$ be a discrete valuation ring, $\Gamma$ a finite group, $R(\Gamma)$ the group ring. We assume that the characteristic of the quotient field $K$ of $R$ does not divide the order of $\Gamma$.

Theorem 14. (i) If the characteristic of the residue class field $k$ of $R$ does not divide the order of $\Gamma$ then $\operatorname{Picent}(R(\Gamma))=1$.

(ii) If $\Gamma$ is a p-group and $k$ is finite (and $K$ is of characteristic 0 ) then Picent $(R(\Gamma))$ is a p-group.

Immediately we have

Corollary. If $R$ is any Dedekind domain, not necessarily local, and if $\Gamma$ is a p-group then $\mathrm{U}_{\mathrm{m}} \operatorname{Picent}\left(R_{\mathrm{m}}(\Gamma)\right)$ is a p-group.

Proof of Theorem 14. (i) $k(\Gamma)$ is separable over $k$. Hence $R(\Gamma)$ is separable over $R$, i.e. is Azumaya over $\operatorname{cent}(R(\Gamma))$, and the latter is a semilocal ring. Thus $\operatorname{Picent}(R(\Gamma))=\operatorname{Picent}(\operatorname{cent}(R(\Gamma)))=1$.

(ii) We may suppose that the residue class field characteristic is $p$. The result follows then by Corollary 3 to Theorem 9, provided we can show that the map $\operatorname{Picent}(R(\Gamma)) \rightarrow \operatorname{Pic}_{R}\left(R(\Gamma) / J(\Gamma)^{2}\right)$ is null, where we shall write $J(\Gamma)$ for the radical of $R(\Gamma)$. As all invertible $R(\Gamma)-R(\Gamma)$-bimodules are free as left $R(\Gamma)$-modules, we may replace here Picent by Outcent. In fact we shall prove more, namely that the map Autcent $(R(\Gamma)) \rightarrow$ Aut $_{R}\left(R(\Gamma) / J(\Gamma)^{2}\right)$ is null. This will be done in a number of steps. This map is of course given by the restriction of a homomorphism

$$
\phi=\phi_{\Gamma}: \operatorname{Aut}_{R}(R(\Gamma)) \rightarrow \operatorname{Aut}_{R}\left(R(\Gamma) / J(\Gamma)^{2}\right)
$$

so that for any $f \in$ Aut $_{R}(R(\Gamma))$ the diagram

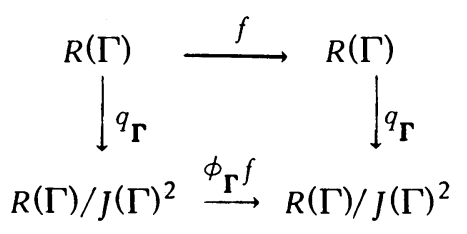

commutes, $q_{\Gamma_{-}}$being the quotient map.

Let now $\bar{\Gamma}$ be the commutator quotient group of $\Gamma$. We shall obtain a unique homomorphism

$$
\psi: \text { Aut }_{R}(R(\Gamma)) \rightarrow \text { Aut }_{R}(R(\bar{\Gamma}))
$$

so that for all $f \in$ Aut $_{R}(R(\Gamma))$ the diagram 


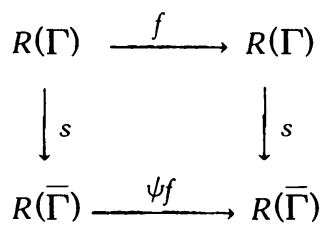

commutes, where $s$ is the quotient map. Then we shall also show that we get a commutative diagram

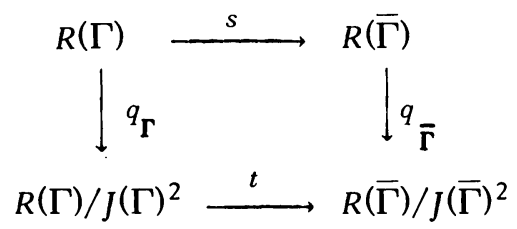

with $t$ an isomorphism. It follows then on identifying via $t$ that $\phi_{\boldsymbol{\Gamma}}=\phi_{\bar{\Gamma}} \circ \psi$. Finally, we shall then prove that $\psi$ maps Autcent $(R(\Gamma))$ into the identity.

First step. The existence and uniqueness of $\psi$ follows from the fact that Ker $s$ is a characteristic ideal. It is the ideal generated by the commutators $x y-y x, \forall x, y \in R(\Gamma)$.

Second step. Let $(\Gamma, \Gamma)$ denote the commutator group of $\Gamma$ and let $L(\Gamma)$ be an additive copy of $\Gamma /(\Gamma, \Gamma) \Gamma^{p}$ with $l(\gamma)$ corresponding to the class of $\gamma$ $\bmod (\Gamma, \Gamma) \Gamma^{p} . L(\Gamma)$ is a vector space over the field $F_{p}$ of $p$ elements. Let $L_{R}(\Gamma)=k \otimes_{F_{p}} L(\Gamma), k=R / m$ the residue class field. $L_{R}(\Gamma)$ is a $R / \mathrm{m}^{2}$-module, via the surjection $R / \mathrm{m}^{2} \rightarrow R / \mathrm{m}$. On the direct sum $R / \mathrm{m}^{2} \oplus L_{R}\left(\Gamma^{\prime}\right)$ we define an algebra-structure by

$$
\left(c_{1}, x_{1}\right)\left(c_{2}, x_{2}\right)=\left(c_{1} c_{2}, c_{1} x_{2}+c_{2} x_{1}\right)
$$

$c_{i} \in R / \mathrm{m}^{2}, x_{i} \in L_{R}(\Gamma)$. Then we can identify $R / \mathrm{m}^{2} \oplus L_{R}(\Gamma)=R(\Gamma) / J(\Gamma)^{2}$ with $q_{\mathbf{\Gamma}}$ being given by $q_{\mathbf{\Gamma}}(\gamma)=\left(1, l_{(\gamma)}\right)\left(1 \in R / \mathrm{m}^{2}\right)$. It is now clear that the required isomorphism $t$, with the above identification, is given by $\left(1, l_{(\gamma)}\right) \mapsto\left(1, l_{(\bar{\gamma})}\right)$, where $\bar{\gamma}=\gamma \bmod (\Gamma, \Gamma)$.

Third step. If $f \in$ Autcent $(R(\Gamma))$, then $f$ fixes the sum $m(\gamma)=\Sigma \gamma^{\prime}$ over the conjugates of an element $\gamma$. Thus $\psi f$ fixes the image of $m(\gamma)$ in $R(\bar{\Gamma})$. But this is just a strictly positive integer multiple of $\bar{\gamma}=\gamma \bmod (\Gamma, \Gamma)$. As $R(\bar{\Gamma})$ is torsion free, $\psi$ f fixes $\bar{\gamma}, \forall \bar{\gamma}$, and so $\psi f=1$, as we had to show.

Next we consider group rings over the ring of integers. In [F] I proved that the kernel $D(\mathbf{Z}(\Gamma)$ ) (cf. $\$ 8$, Theorem 10 for notation)-with $\Gamma$ an Abelian p-group-is itself a $p$-group. Here I shall generalise this to Picard groups of $\mathrm{Z}(\Gamma)$, when $\Gamma$ is an arbitrary finite $p$-group. There is another possible direction in which the Abelian theorem can be extended, namely to the projective class group of $Z(\Gamma)$, 
and this generalisation has in fact been established by I. Reiner and S. Ullom (cf. [RU]). Here I consider the commutative order $A=\operatorname{cent}(\mathrm{Z}(\Gamma))$ and I shall prove

Theorem 15. If $\Gamma$ is a finite p-group then $D(A)$ is a p-group.

Proof. The proof is effectively the same as in the Abelian case. Let $L$ be one of the fields which are direct summands of cent $Q(\Gamma) . L$ is a field of $p^{n}$ th roots of unity over $Q$, and so there is exactly one prime ideal $p$ of the maximal order $M$ of $L$ lying above $p$. Let $U^{(1)}\left(M_{p}\right)$ (note $M_{p}=M_{p}$ ) be the group of units $u \equiv 1(\bmod p)$ of $M_{p}$. Then by Theorem 10 it will suffice to show that $U(M) U^{(1)}\left(M_{\mathfrak{p}}\right)=U\left(M_{\mathfrak{p}}\right)$, or in other words that all prime residue classes of $M / \mathfrak{p}$ are occupied by global units. In fact this is ensured by the Hilbert cyclotomic units.

Finally we consider the group $A_{u t}(R(\Gamma))$, for any commutative ring. Note that the automorphism group $A u t(\Gamma)$ of $\Gamma$ acts on the group $\operatorname{Hom}(\Gamma, U(R))$ of $R$ characters of $\Gamma$, via its action on $\Gamma$. To be explicit let $\phi \in \operatorname{Hom}(\Gamma, U(R))$, $f \in \operatorname{Aut}(\Gamma)$. Then $\phi^{f}(\gamma)=\phi(f(\gamma)), \forall \gamma$. Let $\operatorname{Hom}(\Gamma, U(R)) \cdot \operatorname{Aut}(\Gamma)$ denote the semidirect product of these two groups with respect to the action of Aut $(\Gamma)$ as indicated. Then we get a homomorphism

$$
\theta: \operatorname{Hom}(\Gamma, U(R)) \cdot \text { Aut }(\Gamma) \rightarrow \text { Aut }_{R}(R(\Gamma))
$$

which takes $f \cdot \phi(\phi \in \operatorname{Hom}(\Gamma, U(R)), f \in \operatorname{Aut}(\Gamma))$ into the automorphism $\theta(f \cdot \phi)=$ $[f, \phi]$ with $[f, \phi](\gamma)=f(\gamma) \phi(\gamma)$.

Theorem 16. $\theta$ is injective. If $\Gamma$ is Abelian and $R$ is a ring of algebraic integers then $\theta$ is an isomorphism.

Proof. The first assertion is obvious. The second assertion follows from Higman's Theorem (cf. [GH]).

13. Computations. The dihedral group $\Delta_{2 p}$. Here $p$ is an odd prime and $\Delta_{2 p}=\Delta$ has two generators $\sigma$ and $\tau$, with relations $\tau^{2}=\sigma^{p}=1, \tau \sigma \tau^{-1}=\sigma^{-1}$. The rational group ring $Q(\Delta)$ has three central idempotents, centrally primitive,

$$
e=1-\frac{1}{p} \sum_{i=1}^{p} \sigma^{i}, \quad e_{1}=\frac{1}{2 p} \sum_{i=1}^{p} \sigma^{i}(1+\tau), \quad e_{-1}=\frac{1}{2 p} \sum_{i=1}^{p} \sigma^{i}(1-\tau),
$$

and

$$
e_{ \pm 1} \text { cent } Q(\Delta) \cong Q, \quad e \text { cent } Q(\Delta) \cong L=Q\left(\eta+\eta^{-1}\right)
$$

where $\eta$ is a primitive $p$ th root of unity. These notations will be kept throughout.

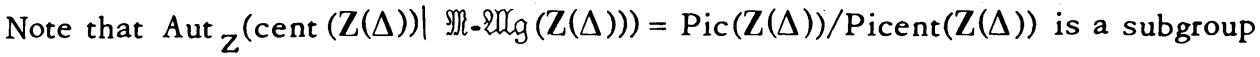
of $A^{A u t}{ }_{Q}(\operatorname{cent}(Q(\Delta)))$. The latter group is the product of a group of order 2 acting 
by permuting the two idempotents $e_{ \pm 1}$, and of the Galois group Gal $(L / Q)$ of $L / Q$ which is cyclic of order $(p-1) / 2$.

We shall need one more notation to state our results. Let $S$ be the integral closure of $\mathbf{Z}$ in $L$. As the maximal order of cent $(Q(\Delta))$ is thus $S \times \mathbf{Z} \times \mathbf{Z}$, we get by Theorem 10 a surjective homomorphism Picent $(\operatorname{cent}(\mathbf{Z}(\Delta))) \rightarrow C(S)$.

Theorem 17. (i) $\operatorname{Pic}(\mathrm{Z}(\Delta)) / \operatorname{Picent}(\mathrm{Z}(\Delta)) \cong \operatorname{Aut}_{Q}{ }^{(\operatorname{cent}(Q(\Delta))) \text { and in fact the }}$ sequence splits.

$$
1 \rightarrow \text { Picent }(\mathrm{Z}(\Delta)) \rightarrow \mathrm{Pic}(\mathrm{Z}(\Delta)) \rightarrow \text { Aut }_{Q}(\text { cent }(Q(\Delta))) \rightarrow 1
$$

(ii) $D(\operatorname{cent}(\mathrm{Z}(\Delta)))$ is cyclic of order $(p-1) / 2$ and the sequence

$$
1 \rightarrow D(\text { cent }(\mathbf{Z}(\Delta))) \rightarrow \text { Picent }(\text { cent }(Z(\Delta))) \rightarrow C(S) \rightarrow 1
$$

splits.

(iii) $\mathrm{T}$ yields an isomorphism

$$
D(\operatorname{cent}(\mathbf{Z}(\Delta))) \times C(S) \cong \operatorname{Picent}(\mathrm{Z}(\Delta))
$$

Remark. One can also determine Outcent $(\mathrm{Z}(\Delta))$. This however requires a result of the forthcoming note [FRU] and will be deferred until then.

Proof of Theorem 17. (i) We know that $\operatorname{Pic}(\mathrm{Z}(\Delta)) / \operatorname{Picent}(\mathrm{Z}(\Delta))$ embeds in ${ }^{A u t} Q_{Q}(\operatorname{cent}(Q(\Delta)))$, while on the other hand, with $\theta$ as in (12.1), the composite map

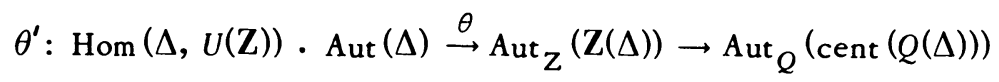

factorises through $\operatorname{Pic}(\mathrm{Z}(\Delta)) / \operatorname{Picent}(\mathrm{Z}(\Delta))$. So we get the isomorphism under (i) if we can show that $\theta^{\prime}$ is surjective. In fact $\operatorname{Aut}_{Q}(\operatorname{cent}(Q(\Delta)))$ is the product of $\mathrm{Gal}(L / Q)$ (which under $\theta^{\prime}$ is the image of the group of automorphisms $\tau \mapsto \tau$, $\sigma \mapsto \sigma^{r}((r, p)=1)$ of $\Delta$ ), and of the transposition $e_{1} \leftrightarrow e_{-1}$ (which under $\theta^{\prime}$ comes

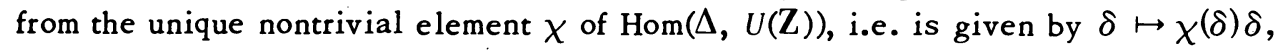
where of course $\chi(\sigma)=1=-\chi(\tau))$. Thus in fact $\theta^{\prime}$ is surjective.

Assuming part (iii) of the Theorem, one observes that $T$ yields an isomorphism of the sequence in (i) with the corresponding sequence for cent $(Z(\Delta))$ in place of $\mathbf{Z}(\Delta)$. But the latter splits (cf. Corollary 1 to Theorem 2).

(ii) Write $A=\operatorname{cent}(\mathbf{Z}(\Delta))$, and use the notation of Theorem 10. Let $M$ be the maximal order of cent $(Q(\Delta))$. Then $e\left(\sigma+\sigma^{-1}\right)$ and $e\left(\sigma^{i}+\sigma^{-i}-\sigma-\sigma^{-1}\right)=\sigma^{i}+$ $\sigma^{-i}-\sigma-\sigma^{-1}$ form a $\mathrm{Z}$-basis of $M e$, which extends to a $\mathrm{Z}$-basis of $M$, by including $e_{1}$ and $e_{-1}$. Localising at $p$, we note that the Jacobson radical $J\left(M_{p}\right)$ of $M_{p}$ lies in $A_{p}$, hence $1+J\left(M_{p}\right) \subset U\left(A_{p}\right)$. Moreover, as is easily verified, $U\left(M_{2}\right)=U\left(A_{2}\right)$. It follows now that $D(A)$ is given by the exactness of the sequence

$$
U\left(A_{p}\right) U(M) \rightarrow U\left(M_{p} / J\left(M_{p}\right)\right) \rightarrow D(A) \rightarrow 1
$$


$M_{p}$ is the direct product of three local $\mathbf{Z}_{p}$-algebras and $A_{p}$ is a local $\mathbf{Z}_{p}$ algebra, and the residue class field in each case is the field of $p$-elements. Therefore $U\left(M_{p} / J\left(M_{p}\right)\right)$ is the product of three copies $V_{1}, V_{-1}$ and $V$ of $U\left(\mathbf{Z}_{p} / p \mathbf{Z}_{p}\right)$ (corresponding to the three idempotents $e_{1}, e_{-1}$ and $e$ ), and the image of $U\left(A_{p}\right)$ is the image of $U\left(\mathbf{Z}_{p}\right)$, i.e. the group $U\left(\mathbf{Z}_{p} / p \mathbf{Z}_{p}\right)$ embedded diagonally in $V_{1} \times$ $V_{-1} \times V$. Finally $U(M)=U_{1} \times U_{-1} \times U$, corresponding to the decomposition $M=M e_{1}+M e_{-1}+M_{e} \cdot U_{1}=\{ \pm 1\}$ maps onto the subgroup of order 2 in $V_{1}$, similarly for $U_{-1}$. Because of the existence of Hilbert cyclotomic units, $U=U(S)$ maps onto the whole of $V$. It now follows that $D(A)$ is indeed cyclic of order $(p-1) / 2$.

- For the splitting of the exact sequence in (ii) we shall define a map $I(A e) \rightarrow$ Picent $(A), I(A e)$ the group of invertible fractional ideals of $A e=$ Me. Let $X \in I(A e)$. Then $X_{p}=b A_{p} e, b=b e$ an invertible element of $(A e \otimes Q)_{p}$. Thus $(1-e+b e) A_{p}$ is an invertible fractional ideal of $A_{p}$ and for all rational primes $q$, other than $p,(1-e) A_{q}+X_{q}$ is an invertible fractional of $(1-e) A_{q}+e A_{q}=A_{q}$. It follows that there is a unique $\bar{X} \in I(A)$ with $\bar{X}_{p}=(1-e+b e) A_{p}, \bar{X}_{q}=(1-e) A_{q}+$ $X_{q}$ all $q \neq p$.

$\bar{X}$ depends on the choice of $b$. We may in fact replace $b$ by $b u, u e=u \in U\left(M_{p} e\right)$. But by what we have already proved, $(1-e+u e)$ is a product of a unit $w \in U\left(A_{p}\right)$ and a global unit $(1-e+v e), v \in U(M e)$. Thus we have to replace $(1-e+b e) A_{p}=$ $\bar{X}_{p}$ by $(1-e+v e) \bar{X}_{p}$. For all $q \neq p, 1-e+v e \in U\left(A_{p}\right)$, hence $\bar{X}_{q}=(1-e+v e) \bar{X}_{q}$. Therefore starting with $b u$ in place of $b$ we end up with $(1-e+v e) \bar{X}$ in place of $\bar{X}$. Hence $X \mapsto \operatorname{cl}(\bar{X}) \in \operatorname{Picent}(A)$ is a uniquely defined map. One verifies easily that it is multiplicative and that the homomorphism Picent $(A) \rightarrow \operatorname{Picent}(A e) \cong$ $C(S)$ takes $\operatorname{cl}(\bar{X})$ into $\operatorname{cl}(X)$. Finally it is obvious that if $X=a A e, a=a e$ invertible in $A e \otimes Q$, then $\bar{X}=((1-e)+a e) A$ is principal. Thus the map $I(A e) \rightarrow$ $\operatorname{Picent}(A)$ yields a homomorphism $C(S) \cong \operatorname{Picent}(A e) \rightarrow \operatorname{Picent}(A)$ splitting the exact sequence in (ii).

Finally we shall show that

$$
\text { Picent }\left(Z_{2}(\Delta)\right)=1
$$

and

$$
\text { Picent }\left(Z_{p}(\Delta)\right)=1
$$

By Theorem 14(i), (ii) and by the localisation theorem we then have Picent $(\mathrm{Z}(\Delta)$ ) $\cong C(S) \times D(A)$.

Let in the sequel $\Pi$ denote the group of order 2 and let $E=Q(\eta)$ be the field of $p$ th roots of unity and $T=\mathrm{Z}[\eta]$ its ring of integers. We shall write $\overparen{E(I I)}$ and $\overparen{T(\Pi)}$ for the twisted group rings or trivial crossed product, the generator $\pi$ of $\Pi$ acting by $\eta^{\pi}=\eta^{-1}$. Then 


$$
Q(\Delta) \cong Q(\Pi) \times \overparen{E(\Pi)}
$$

with $Q(\Delta)\left(e_{1}+e_{-1}\right) \cong Q(\Pi)$ via $\left(e_{1}+e_{-1}\right) \tau \mapsto \pi$ and $Q(\Delta) e \cong \overparen{E(\Pi)}$ via $e \sigma \mapsto \eta$, er $\mapsto \pi$. It follows that

$$
\mathrm{Z}_{2}(\Delta) \cong \mathrm{Z}_{2}(\Pi) \times \widetilde{T_{2}(\Pi)}
$$

One knows that Picent $\left(Z_{2}(\Pi)\right)=1$ (see e.g. $\left.[F]\right)$. Also $\overparen{T_{2}(\Pi)}=\operatorname{End}_{s_{2}}\left(T_{2}\right)$, hence $\operatorname{Picent}\left(\overparen{\left.T_{2}(\Pi)\right)}\right)=1$. Thus (13.1) does hold.

For (13.2) we need a rather more sophisticated fibre product technique. We shall not give here a general treatment but stick to the relevant special case. The relevant commutative square is

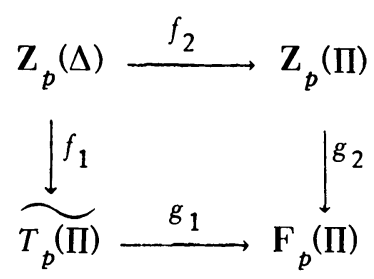

where $F_{p}$ is the field of $p$-elements, and the maps $g_{1}, g_{2}$ are residue class maps modulo the ideal generated by the unique maximal ideals in $\mathrm{Z}_{p}$ and in $T_{p}$ respectively. The maps from $Z_{p}(\Delta)$ comes from (13.3).

First of all observe that, as every invertible $\mathrm{Z}_{p}(\Delta)-\mathrm{Z}_{p}(\Delta)$-bimodule is free, we may replace Picent by Outcent. We shall then show below that

$$
\text { Outcent } \left.\left(\mathbf{Z}_{p}(\Delta)\right) \cong \text { Outcent } \overparen{\left(T_{p}(\Pi)\right.}, g_{1}\right)
$$

where the group on the right is the subgroup of Outcent $\left(\widetilde{T_{p}(\Pi)}\right)$ of classes of automorphisms $\alpha$ with $g_{1} \circ \alpha=g_{1}$.

We shall view $\overparen{T_{p}(\Pi)}$ as embedded in the 2 by 2 matrix rings End $s_{p}\left(T_{p}\right)$ and End $_{L_{p}}\left(E_{p}\right)$ over $S_{p}$ and $L_{p}$ respectively, with respect to the basis 1 and $\left(\eta-\eta^{-1}\right) / 2=$ $\mu$, with $\mu$ and $\mu^{2}$ prime elements in $T_{p}$ and in $S_{p}$ respectively. Then $T_{p}(\Pi)$, as algebra over $S_{p}$, is generated by the matrices

$$
\begin{aligned}
& m_{\mu}=\left(\begin{array}{ll}
0 & 1 \\
\mu^{2} & 0
\end{array}\right) \quad(\text { multiplication by } \mu), \\
& m_{\pi}=\left(\begin{array}{rr}
1 & 0 \\
0 & -1
\end{array}\right) \quad(\text { action of } \pi),
\end{aligned}
$$

and the se two matrices together with the identity matrix $m_{1}$ and the product $m_{\pi} \cdot m_{\mu}$

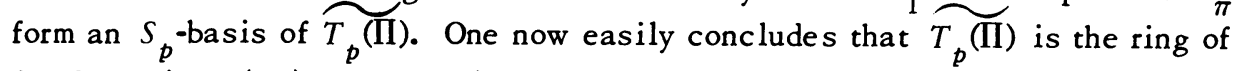
$2 \times 2$ matrices $\left(a_{i j}\right)$ over $S_{p}$, with $a_{21}$ lying in the maximal ideal. (This is in fact 
a special case of a general result on the twisted group rings of tamely ramified extensions). To put it differently, $T_{p}(\mathrm{II})$ is the intersection of the subrings End $_{S_{p}}\left(T_{p}\right)$ and End $S_{p}\left(\mu T_{p}\right)$ of End $L_{p}\left(E_{p}\right)$, and these are the only two maximal orders containing it. Thus any element of the normaliser $N\left(T_{p}(\Pi)\right)$ either normalises End $_{S_{p}}\left(T_{p}\right)$, i.e. is a unit of the latter ring times a central element, or it is the product of such a normaliser of $\operatorname{End}_{S_{p}}\left(T_{p}\right)$ with a matrix which interchanges the two maximal orders, e.g. with $m_{\mu}$. One now verifies firstly that

$$
U\left(\operatorname{End}_{S_{p}}\left(T_{p}\right)\right) \cap N\left(\widetilde{T_{p}(\Pi)}\right)=U\left(\widetilde{\left.T_{p}(\Pi)\right)},\right.
$$

e.g. by explicit computation. Thus every central automorphism $\alpha$ of $\overparen{T_{p}(\Pi)}$ is induced by conjugation with a matrix $u m_{\mu}^{s}$, where $u \in U\left(T_{p}(\Pi)\right), s=0$ or 1 . If $s=0$ then indeed $g_{1} \circ \alpha=g_{1}$. But $m_{\mu}^{-1} m_{\pi} m_{\mu}=-m_{\pi}$, and so if $s=1$ then $g_{1} \circ \alpha \neq g_{1}$. Hence finally

$$
\text { Outcent } \left.\widetilde{\left(T_{p}(\Pi)\right.}, g_{1}\right)=1
$$

Given (13.5) this finally yields (13.2) and completes the proof.

It remains to establish a lemma on a special fibre product and to deduce (13.5) from it. We consider a commutative diagram of rings, with $g_{1}$ and $g_{2}$ surjections,

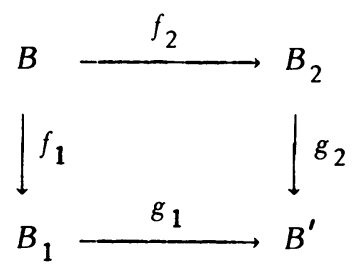

where $B$ and $B_{1} \times B_{2}$ are orders over a Dedekind domain $R$, both spanning the same separable algebra over the quotient field $K$. We assume that (13.6) is a fibre product diagram, i.e. that $B$ is the subring of $B_{1} \times B_{2}$ of pairs $\left(b_{1}, b_{2}\right)$ with $g_{1} b_{1}=$ $g_{2} b_{2}$. We moreover assume that $B_{2}$ and $B^{\prime}$ are commutative. Then we have an is omorphism

$$
t: \text { Autcent }(B) \cong \text { Autcent }\left(B_{1}, g_{1}\right)
$$

where the group on the right consists of those automorphisms $\alpha$ with $g_{1} \circ \alpha=g_{1}$. There results a surjective homomorphism

$$
\bar{t}: \text { Outcent }(B) \rightarrow \text { Outcent }\left(B_{1}, g_{1}\right)
$$

the group on the right viewed as embedded in Outcent $\left(B_{1}\right)$. 
Lemma D. $\operatorname{Ker} \bar{t} \cong g_{1}\left(U\left(B_{1}\right)\right) / g_{1}\left(U\left(\operatorname{cent}\left(B_{1}\right)\right)\right) \cdot\left(g_{1}\left(U\left(B_{1}\right)\right) \cap g_{2}\left(U\left(B_{2}\right)\right)\right)$.

Proof. With $\alpha \in$ Autcent $(B)$, where $t \alpha$ is an inner automorphism of $B_{1}$, say induced by $u_{1}$, associate the class $\overline{g_{1}\left(u_{1}\right)}$ of $g_{1}\left(u_{1}\right)$ in the group appearing on the right in the lemma. This class is clearly independent of the particular choice of $u_{1}$ in $U\left(B_{1}\right)$. Moreover, as

$$
g_{1}\left(U\left(B_{1}\right)\right) \cap g_{2}\left(U\left(B_{2}\right)\right) \supset g_{1} f_{1}(U(B))
$$

$\overline{g_{1}\left(u_{1}\right)}$ only depends on the class $\operatorname{cl}(\alpha)$ of $\alpha$ in Outcent $(B)$. We thus get a welldefined map $\operatorname{cl}(\alpha) \rightarrow \overline{g_{1}\left(u_{1}\right)}$, which is a homomorphism $r$ of $\operatorname{Ker} \bar{t}$ into the group on the right-hand side in Lemma D.

$r$ is surjective. For, every inner automorphism $\alpha$ of $B_{1}$ satisfies $g_{1} \circ \alpha=g_{1}$, as $B^{\prime}$ is commutative. $r$ is also injective. For suppose that $g_{1}\left(u_{1}\right)=g_{2}\left(u_{2}\right) g_{1}(v)$, $u_{2} \in U\left(B_{2}\right), v \in U\left(\operatorname{cent}\left(B_{1}\right)\right)$. Then the inner automorphism $\alpha_{1}$ of $B_{1}$, induced by $u_{1}$, satisfies $\alpha_{1}=t \alpha$, where $\alpha$ is the inner automorphism of $B$ induced by $\left(u_{1} v^{-1}, u_{2}\right)$.

It remains to deduce (13.5) from the lemma. The conditions are certainly satisfied, with $(13.6)=(13.4)$. Moreover as $Z_{p}(I I)$ is a complete semilocal ring and $F_{p}(\Pi)$ the residue class ring modulo its radical, $g_{2}$ induces a surjection on the unit groups, i.e. in the notation of Lemma $\mathrm{D}, g_{2}\left(U\left(B_{2}\right)\right) \supset g_{1}\left(U\left(B_{1}\right)\right)$. Therefore $\bar{t}$ is an isomorphism.

14. Computations. The dihedral group and the quaternion group of order 8 . Let $\Gamma$ be one of the two groups in the title of this section, and write $A=$ cent $(\mathrm{Z}(\Gamma))$. We shall prove

Theorem 18. $D(A) \cong \operatorname{Picent}(A) \cong \operatorname{Picent}(Z(\Gamma))$ is of order 2. Also Outcent $(\mathrm{Z}(\Gamma)$ ) is of order $2, \Gamma$ the dibedral group; Outcent $(\mathrm{Z}(\Gamma))=1, \Gamma$ the quaternion group.

Remark. The fact that Picent $(\mathrm{Z}(\Gamma)) / \operatorname{Outcent}(\mathrm{Z}(\Gamma))$ is of order 2 in the quaternion case follows from a result of Martinet (cf. $[M])$.

Proof of Theorem 18. We first evaluate card $D(A)$, using the formulae of Theorem 10 .

We describe $\Gamma$ by generators $\sigma$ and $\tau$ and relations $\sigma^{4}=1, \tau \sigma \tau^{-1}=\sigma^{-1}$ and $\tau^{2}=1$ ( $\Gamma$ the dihedral group) or $\tau^{2}=\sigma^{2}$ ( $\Gamma$ the quaternion group). The central, centrally primitive idempotents of $Q(\Gamma)$ are

$$
e=\frac{1}{2}\left(1-\sigma^{2}\right), \quad e_{\chi}=\frac{1}{8} \sum_{\gamma \in \Gamma} \gamma \chi(\gamma)
$$

where $\chi$ runs through the elements of $\operatorname{Hom}(\Gamma, U(Z))$-there are four of these. The corresponding simple components of cent $(Q(\Gamma))$ are all isomorphic to $Q$. Thus the 
maximal order $M$ of cent $(Q(\Gamma))$ is the direct sum of five copies of $\mathbf{Z}$, and on localising at 2 we get $U\left(M_{2} / J\left(M_{2}\right)\right)=U\left(A_{2} / J\left(A_{2}\right)\right)=1$.

Now $J\left(M_{2}\right)=2 M_{2}$. Writing $N$ for the subgroup of $2 M$ generated by $2 e$ and $8 e_{\chi}$, for all $\chi$, we note that $N \subset A$, in fact $N_{2} \subset J\left(A_{2}\right)$, and that $\left[J\left(M_{2}\right): N_{2}\right]=4^{4}$. Hence $\left[J\left(M_{2}\right): J\left(A_{2}\right)\right]=4^{4} /\left[J\left(A_{2}\right): N_{2}\right]$. Now $J\left(A_{2}\right)$ is generated freely by $2 e$ and by the elements $c_{\gamma}=\gamma+\gamma \sigma^{2}(\gamma=1, \sigma, \tau, \sigma \tau)$. Expressing the free generators $2 e, 8 e_{\mathbf{x}}$ of $N$ in terms of those of $J\left(A_{2}\right)$ we conclude that $\left[J\left(A_{2}\right): N_{2}\right]=16$. Hence $\left[U\left(M_{2}\right): U\left(A_{2}\right)\right]=\left[J\left(M_{2}\right): J\left(A_{2}\right)\right]=4^{4} / 16=16$. Moreover $\operatorname{card}(U(M))=2^{5}$, namely $U(M)$ consists of the elements

$$
s e+\sum_{\chi} s_{\chi} e_{x}, \quad s \text { and } s_{\chi}= \pm 1
$$

Checking which of those lie in $A$, we conclude that $U(A)$ consists of \pm 1 and $\pm \sigma^{2}$. Hence card $D(A)=16 \cdot 4 / 32=2$. Trivially also $D(A) \cong \operatorname{Picent}(A)$.

To show that Picent $(A) \cong \operatorname{Picent}(Z(\Gamma))$ we have, by Theorem 6 and Theorem 14 , to prove that $\operatorname{Picent}\left(Z_{2}(\Gamma)\right)=1$, or equivalently that

$$
\operatorname{Outcent}\left(Z_{2}(\Gamma)\right)=1
$$

For this and also for the computation of Outcent $(Z(\Gamma))$ we shall use a fibre product diagr am

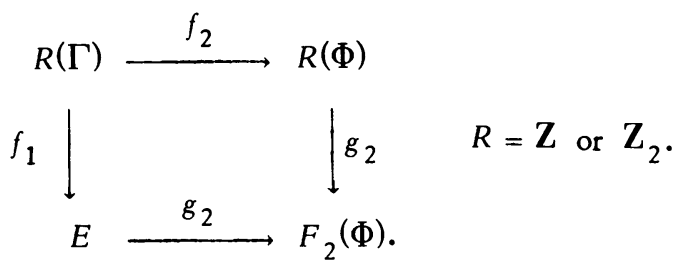

Here $\Phi$ is the Vierer group $\Gamma /\left\{\sigma^{2}\right\}$, with $f_{2}$ as quotient map. $F_{2}$ is the field of 2 elements. If $\Gamma$ is the dihedral group then $E$ is the twisted group ring (trivial crossed product) of the group $\Pi$ of order 2 over $\mathbf{Z}[i]$, or $\mathbf{Z}_{2}[i]$ respectively, where $i^{2}=-1$, and where the generator $\pi$ of $\Pi$ acts by $i \mapsto-i$. If $\Gamma$ is the quaternion group, then $E$ is the standard quaternion algebra over $\mathbf{Z}$, or over $\mathrm{Z}_{2}$ respectively, with basis $1, i, j, k$ where $i^{2}=j^{2}=k^{2}=-1, i j=-j i=k$. In all cases $g_{1}$ and $g_{2}$ are residue class maps mod 2 .

The hypotheses of Lemma D are now seen to hold in all cases. We shall show that

$$
\text { Outcent }(R(\Gamma)) \cong \text { Outcent }\left(E, g_{1}\right) \text {. }
$$

if $R=\mathrm{Z}_{2}$, or if $R=\mathbf{Z}$ and $\Gamma$ is the quaternion group. In the case $R=Z_{2}$, the map $g_{2}$ induces a surjection of the groups of units, as $F_{2}(\Phi)$ is the quotient of 
the semilocal ring $\mathrm{Z}_{2}(\Phi)$ modulo an ideal inside the radical. Thus (14.3) holds by Lemma D. If $R=\mathrm{Z}$ and $\Gamma$ is the quaternion group then the only units of $E$ are $\pm 1, \pm i, \pm j$ and $\pm k$ and the se are mapped onto $\Phi \subset g_{2}(U(\mathbf{Z}(\Phi)))$ by $g_{1}$. Hence again (14.3) holds.

We shall first complete the quaternion case. Now $E$ will stand for the global quaternion ring $\mathrm{Z}[i, j, k]$, and we shall write $H=E \otimes_{Z} Q$. Let $P_{2}$ be the maximal ideal in the unique maximal order $M_{2}$ of $H_{2}$ (the subscript denotes completion at 2). Both $P_{2}$ and $M_{2}$ are normalised by the whole of $U\left(H_{2}\right)$, hence so is $E_{2}=$ $\mathrm{Z}_{2}+P_{2}$ (sum of submodules of $M_{2}$ ). Every element of $U\left(H_{2}\right)$ has a representation in the form

$$
v u(1+i)^{s}, \quad s=0 \text { or } 1, u \in U\left(M_{2}\right), v \in U\left(Q_{2}\right),
$$

and the automorphism it induces only depends on $u$ and $s$, and the corresponding element of Outcent $\left(E_{2}\right)$ only on $u \bmod U\left(E_{2}\right)$ and $s$. Now $U\left(M_{2}\right) / U\left(E_{2}\right) \cong$ $U\left(M_{2} / P_{2}\right)$ is of order 3. Thus Outcent $\left(E_{2}\right)$ is of order 6, while, by (14.3) and Theorem 14(ii), Outcent $\left(E_{2}, g_{1}\right)$ has 2-power order, and thus is of order 1 or 2. In other words any automorphism $\alpha$ whose class falls into Outcent $\left(E_{2}, g_{1}\right)$ is given by an element $u(1+i)^{s}, s=0$ or $1, u \in U\left(E_{2}\right)$. But as $(1+i) j \equiv j(1+i)$ (mod 2) we must have $s=0$. Thus in fact $\operatorname{Outcent}\left(E_{2}, g_{1}\right)=1$, hence by (14.3) we have (14.1) in this case.

Still keeping to the quaternion case, let $M$ be the unique maximal order of $H$, containing $E$, with local components $M_{p}=E_{p}$ when $p \neq 2$. By Theorem 12, Picent $(M)$ is of order 2. As $(1+i) \in U\left(M_{p}\right)$, all $p \neq 2$, and $(1+i) \in N\left(M_{2}\right)$, it follows that $(1+i)$ induces an automorphism of $M$, which is not inner. As Outcent $(M) \subset \operatorname{Picent}(M)$ is of order at most 2 , this is essentially the only outer automorphism of $M$. On the other hand any normaliser of $E$ also normalises $M$, hence is of form

$$
v u(1+i)^{s}, \quad s=0 \text { or } 1, \quad u \in U(M), \quad v \in U(Q) .
$$

Now one proceeds just as in the local case, or appeals to what has been proved in the local case to deduce that $\operatorname{Outcent}\left(E, g_{1}\right)=1$. By $(14.3)$, Outcent $(Z(\Gamma))=1$.

From now let $\Gamma$ be the dihedral group and let now $E$ be the twisted group ring. of $\Pi$ over $\mathrm{Z}[i]$. View $E$ as embedded in the matrix rings $\operatorname{End}_{\mathbf{Z}}(\mathbf{Z}[i])$ and End $Q^{(Q(i))}$ defined with respect to the basis $1, i$. A basis of $E$ over $Z$ is given by the identity matrix $m_{1}$,

$$
m_{i}=\left(\begin{array}{rr}
0 & 1 \\
-1 & 0
\end{array}\right), \quad m_{\pi}=\left(\begin{array}{rr}
1 & 0 \\
0 & -1
\end{array}\right)
$$

and $m_{i} \cdot m_{\pi}$. Moreover under $g_{1}$ these four matrices are mapped precisely into $\Phi$. Now 


$$
m_{1}+m_{i}+m_{\pi}=\left(\begin{array}{rr}
2 & 1 \\
-1 & 0
\end{array}\right)
$$

is a unit of $E$, whose image in $F_{2}(\Phi)$ does not lie in $\Phi$, hence by Higman's Theorem (cf. $[\mathbf{G H}]$ ) does not lie in $g_{2}(U(\mathrm{Z}(\Phi)))$. By Lemma $\mathrm{D}, \operatorname{Ker} \bar{t} \neq 1$, hence

$$
\text { Outcent }(\mathbf{Z}(\Gamma)) \neq 1, \quad \Gamma \text { dihedral. }
$$

Now complete at $p=2$. Then $E_{2}$ is contained in precisely two maximal orders, namely the $\mathbf{Z}_{2}$-endomorphism rings of $\mathbf{Z}_{2}[i]$ and of its ideal $(1+i)$, and the se two orders are interchanged by conjugation by $m_{1}+m_{i}$. Thus modulo central elements any normaliser of $E_{2}$ can be written in the form $u\left(m_{1}+m_{i}\right)^{s}$, $s=0$ or 1 , and $u=\left(a_{k l}\right)$ an invertible matrix over $Z_{2}$. Now the matrices $\left(b_{k l}\right)$ in $E_{2}$ are characterised by

$$
b_{11}+b_{22} \equiv b_{12}+b_{21} \equiv 0 \quad(\bmod 2) .
$$

Applying this criterion to the conjugate

$$
m_{i}^{\prime}=\left(u\left(m_{1}+m_{i}\right)^{s}\right) \cdot m_{i} \cdot\left(u\left(m_{1}+m_{i}\right)^{s}\right)^{-1}
$$

of $m_{i}$, we get, as condition for $m_{i}^{\prime}$ to lie in $E_{2}$, that $\Sigma_{k, l} a_{k l}^{2}=0(\bmod 2)$. As $a_{11} a_{22}-a_{12} a_{21} \in U\left(\mathbf{Z}_{2}\right)$ this implies that $a_{11}^{2}+a_{22}^{2} \equiv a_{12}^{2}+a_{21}^{2} \equiv 0(\bmod 2)$, i.e. that $u \in U\left(E_{2}\right)$. But $m_{\pi} \equiv\left(u\left(m_{1}+m_{i}\right)^{s}\right) \cdot m_{\pi} \cdot\left(u\left(m_{1}+m_{i}\right)^{s}\right)^{-1}(\bmod 2) \mathrm{im}$ plies $s=0$. Thus in fact Outcent $\left(E_{2}, g_{1}\right)=1$, hence by $(14.3)$ Outcent $\left(Z_{2}(\Gamma)\right)=1$ as was required. Thus Picent $(Z(\Gamma))$ is of order 2, and hence by (14.4) Outcent $(Z(\Gamma))$ is of order 2 .

\section{LITERATURE LIST}

[B1] H. Bass, Lectures on topics in algebraic K-theory, Tata Inst. of Fund. Res. Lectures on Math., no. 41, Tata Institute of Fundamental Research, Bombay, 1967. MR 43 \#4885.

[B2] —-, Algebraic K-theory, Benjamin, New York, 1968. MR 40 \#2736.

[DE] M. Deuring, Algebren, Springer, Berlin, 1935; English transl., Chelsea, New York, 1948.

[F] A. Fröhlich, On the classgroup of integral grouprings of finite Abelian groups, Mathematika 16 (1969), 143-152. MR 41 \#5512.

[FRU] A. Fröhlich, I. Reiner and S. Ullom, Picard groups and class groups (to be published).

[DH] D. G. Higman, On orders in separable algebras, Canad. J. Math 7 (1955), 509515. MR 19, 527.

[GH] G. Higman, The units of group-rings, Proc. London Math. Soc. (2) 46 (1940), 231-248. MR 2, 5 .

[M] J. Martinet, Modules sur l'algèbre du group quaternionien, Ann. Sci. École Norm. Sup. (4) 4 (1971), 399-408. 
[R] I. Reiner, A survey of integral representation theory, Bull. Amer. Math. Soc. 76 (1970), 159-227. MR $40 \# 7302$.

[RU] I. Reiner and S. Ullom, Class groups of integral group rings, Trans. Amer. Math. Soc. 170 (1972), 1-30.

[RHD] K. W. Roggenkamp and V. Huber-Dyson, Lattices over orders. I, Lecture Notes in Math., vol. 115, Springer-Verlag, Berlin and New York, 1970. MR 44 \#247a.

[RZ] A. Rosenberg and D. Zelinsky, Automorphisms of separable algebras, Pacific J. Math. 11 (1961), 1109-1117. MR $26 \# 6215$.

[ST] J. R. Strooker, Faithfully projective modules and clean algebras, Dissertation, University of Utrecht, Utrecht, 1965, Groen \& Zoon, N. V., Leiden, 1965. MR 36 \#206.

[Sw] R. G. Swan, K-theory of finite groups and orders, Lecture Notes in Math., vol. 149, Springer-Verlag, Berlin and New York, 1970.

DEPARTMENT OF MATHEMATICS, KING'S COLLEGE, LONDON, ENGLAND 